\title{
A characterization of convex calibrable sets in $\mathbb{R}^{N}$
}

\section{F. Alter ${ }^{*}$, V. Caselles ${ }^{\dagger}$, A. Chambolle ${ }^{\ddagger}$}

16th December 2003

\begin{abstract}
The main purpose of this paper is to characterize the calibrability of bounded convex sets in $\mathbb{R}^{N}$ by the mean curvature of its boundary, extending the known analogous result in dimension 2 . As a by-product of our analysis we prove that any bounded convex set $C$ of class $C^{1,1}$ has a convex calibrable set $K$ in its interior, and and for any volume $V \in[|K|,|C|]$ the solution of the perimeter minimizing problem with fixed volume $V$ in the class of sets contained in $C$ is a convex set. As a consequence we describe the evolution of convex sets in $\mathbb{R}^{N}$ by the minimizing total variation flow.
\end{abstract}

Key words: Calibrable sets, convex sets, mean curvature, Total Variation.

AMS (MOS) subject classification: 35K65, 35K55.

\section{Introduction}

The characterization of convex calibrable sets in the plane in terms of the mean curvature of its boundary was proved in [27]. Indeed, given a bounded convex set $C \subseteq \mathbb{R}^{2}$ of class $C^{1,1}$, the following statements are equivalent $([27],[22])$

(a) $C$ is a solution of the problem

$$
\min _{X \subseteq C} P(X)-\lambda_{C}|X| \quad \text { where } \lambda_{C}=\frac{P(C)}{|C|} .
$$

(b) There is a vector field $z \in L^{\infty}\left(\mathbb{R}^{2}, \mathbb{R}^{2}\right),\|z\|_{\infty} \leq 1$ such that

$$
\begin{aligned}
& -\operatorname{div} z=\lambda_{C} \quad \text { in } C \\
& z \cdot \nu^{C}=-1 \quad \text { a.e. in } \partial C
\end{aligned}
$$

where $\nu^{C}$ denotes the outer unit normal to $\partial C$.

(c) $\operatorname{ess} \sup _{p \in \partial C} k_{\partial C}(p) \leq \lambda_{C}$.

This result implies the existence of solutions of the capillary problem in the absence of gravity for any contact angle $\gamma \in\left(0, \frac{\pi}{2}\right]$ (even for $\gamma=0,[27]$ ). This result was also improved (proving that $(a)$ implies that $C$ must be convex and of class $C^{1,1}$ ) and used in [11] to describe those sets which evolve by the minimizing Total Variation flow

$$
\left.\frac{\partial u}{\partial t}=\operatorname{div}\left(\frac{D u}{|D u|}\right) \quad \text { in } Q_{T}:=\right] 0, T\left[\times \mathbb{R}^{2},\right.
$$

${ }^{*}$ CMLA, ENS Cachan, 61 Av. du Président Wilson, 94235 Cachan Cedex, France, email: Francois.Alter@ens.fr

${ }^{\dagger}$ Departament de Tecnologia, Universitat Pompeu-Fabra, Passeig de Circumvalació 8, 08003 Barcelona, Spain, email: vicent.caselles@upf.edu

${ }^{\ddagger}$ CEREMADE, CNRS UMR 7534, Université Paris-Dauphine, 75775, Paris Cedex 16, France, email: antonin@ceremade.dauphine.fr 
without distortion of its boundary, i.e., the sets $\Omega \subseteq \mathbb{R}^{2}$ whose solution of $(2)$ with $u(0, x)=\chi_{\Omega}(x)$ is given by $u(t, x)=\left(1-\frac{P(\Omega)}{|\Omega|} t\right)^{+} \chi_{\Omega}$. Later, in [1], using these results the authors described the evolution by (2) of any convex set in the plane.

Let us mention that the calibrability of plane convex sets in the case of Finsler metrics has been extensively studied by G. Bellettini, M. Novaga, and E. Paolini [14], (see also [13]) in connection with the problem of facet breaking of crystals which evolve under anisotropic mean curvature flow. The development of facets in crystal evolution has also been considered in [25], [26] using a variational approach.

The main purpose of this paper is to extend the above result to the case of bounded convex sets of class $C^{1,1}$ in $\mathbb{R}^{N}$. The main tools of our analysis will be the study of the variational problems

$$
\min _{X \subseteq C} P(X)-\lambda|X|, \quad \lambda>0,
$$

and its relation with the associated variational problem

$$
\min _{u \in B V\left(\mathbb{R}^{N}\right) \cap L^{2}\left(\mathbb{R}^{N}\right)} \int_{\mathbb{R}^{N}}|D u|+\frac{\mu}{2} \int_{\mathbb{R}^{N}}\left(u-\chi_{C}\right)^{2} d x \quad(\mu>0) .
$$

It turns out that the level sets of the solution of (4) embed the solutions of (3) for the values of $\lambda \in[0, \mu]$. This will be exploited to give the characterization of the calibrability of $C$ in terms of the mean curvature of its boundary. Indeed, we shall characterize for which values of $\lambda$ the solution of (3) coincides with $C$. Indeed, the set of such $\lambda$ coincides with the interval $\left[\max \left(\lambda_{C},(N-1)\left\|\mathbf{H}_{C}\right\|_{\infty}\right), \infty\right)$, where $\mathbf{H}_{C}(x)$ denotes the mean curvature of $\partial C$ at the point $x$. As an interesting by-product of our analysis we shall prove that the solutions of (3) are convex sets. Since (3) can be considered as the functional obtained by applying the Lagrange multiplier method to the area minimizing problem

$$
\min _{X \subseteq C,|X|=V} P(X)
$$

where $0<V<|C|$, we shall obtain that, for some range of volumes $V$, the solutions of this isoperimetric problem with fixed volume $V$ in $C$ are convex sets. The range of values of $V$ for which the above result holds is $[|K|,|C|]$ where $K$ is a convex calibrable set contained in $C$. A positive answer to this problem in the case of convex sets $C$ containing a ball $B$ such that $\partial B \cap \partial C$ is a large circle of $B$ has been given by E. Stredulinsky and W.P. Ziemer in [41]. There is also a positive answer to this problem when $C$ is a rotationally symmetric convex body [36].

As a consequence of the characterization of convex calibrable sets in $\mathbb{R}^{N}$ we may describe its explicit evolution by (2) by means of the same formula than in the $2 D$ case, i.e.,

$$
u(t, x)=\left(1-\frac{P(C)}{|C|} t\right)^{+} \chi_{C} .
$$

We can also describe the evolution of any convex set (more generally, of unions of them which satisfy some additional condition) in $\mathbb{R}^{N}$.

Let us describe the plan of the paper. In Section 2 we collect some preliminaries about functions of bounded variation, Green's formula, the subdifferential of the total variation in $\mathbb{R}^{N}$, calibrable sets, and the corresponding Dirichlet problem for total variation in a bounded domain in $\mathbb{R}^{N}$. Section 3 is devoted to the characterization of calibrability of convex sets in terms of the mean curvature of its boundary. For that we first study the basic properties of the minimizers of problems 3 and 4 . In Section 4 we prove the convexity of the solutions of 5 when $V \in[|K|,|C|]$ where $K$ is a convex calibrable set contained in $C$. Section 5 is devoted to the description of the evolution of convex by the minimizing TV flow (2). We also describe the evolution of several convex sets satisfying an additional condition meaning that they are sufficiently far apart to have no interaction between them. 


\section{Preliminaries}

\section{1 $B V$ functions and sets of finite perimeter}

Let $Q$ be an open subset of $\mathbb{R}^{N}$. A function $u \in L^{1}(Q)$ whose gradient $D u$ in the sense of distributions is a (vector valued) Radon measure with finite total variation in $Q$ is called a function of bounded variation. The class of such functions will be denoted by $B V(Q)$. The total variation of $D u$ on $Q$ turns out to be

$$
\sup \left\{\int_{Q} u \operatorname{div} z d x: z \in C_{0}^{\infty}\left(Q ; \mathbb{R}^{N}\right),\|z\|_{L^{\infty}(Q)}:=\underset{x \in Q}{\operatorname{ess} \sup }|z(x)| \leq 1\right\},
$$

(where for a vector $v=\left(v_{1}, \ldots, v_{N}\right) \in \mathbb{R}^{N}$ we set $|v|^{2}:=\sum_{i=1}^{N} v_{i}^{2}$ ) and will be denoted by $|D u|(Q)$ or by $\int_{Q}|D u|$. It turns out that the map $u \rightarrow|D u|(Q)$ is $L_{\text {loc }}^{1}(Q)$-lower semicontinuous. $B V(Q)$ is a Banach space when endowed with the norm $\int_{Q}|u| d x+|D u|(Q)$. We recall that $B V\left(\mathbb{R}^{N}\right) \subseteq L^{N /(N-1)}\left(\mathbb{R}^{N}\right)$. The total variation of $u$ on a Borel set $B \subseteq Q$ is defined as $\inf \{|D u|(A): A$ open , $B \subseteq A \subseteq Q\}$. We denote by $B V_{\text {loc }}(Q)$ the space of functions $w \in L_{\text {loc }}^{1}(Q)$ such that $w \varphi \in B V(Q)$ for all $\varphi \in C_{0}^{\infty}(Q)$. For results and informations on functions of bounded variation we refer to [4], [21].

A measurable set $E \subseteq \mathbb{R}^{N}$ is said to be of finite perimeter in $Q$ if (6) is finite when $u$ is substituted with the characteristic function $\chi_{E}$ of $E$. The perimeter of $E$ in $Q$ is defined as $P(E, Q):=\left|D \chi_{E}\right|(Q)$, and $P(E, Q)=P\left(\mathbb{R}^{N} \backslash E, Q\right)$. We shall use the notation $P(E):=P\left(E, \mathbb{R}^{N}\right)$. For sets of finite perimeter $E$ one can define the essential boundary $\partial^{*} E$, which is countably $(N-1)$ rectifiable with finite $\mathcal{H}^{N-1}$ measure, and compute the outer unit normal $\nu^{E}(x)$ at $\mathcal{H}^{N-1}$ almost all points $x$ of $\partial^{*} E$, where $\mathcal{H}^{N-1}$ is the $(N-1)$ dimensional Hausdorff measure. Moreover, $\left|D \chi_{E}\right|$ coincides with the restriction of $\mathcal{H}^{N-1}$ to $\partial^{*} E$.

If $\mu$ is a (possibly vector valued) Radon measure and $f$ is a Borel function, the integration of $f$ with respect to $\mu$ will be denoted by $\int f d \mu$. When $\mu$ is the Lebesgue measure, the symbol $d x$ will be often omitted.

If $E$ is a subset of $\mathbb{R}^{N}$ of class $\mathcal{C}^{1,1}$, we denote by $\mathbf{H}_{E}$ the $\left(\mathcal{H}^{N-1}\right.$-almost everywhere defined) mean curvature of $\partial E$, nonnegative for convex sets. As observed in [12], the following result can be proved as in [35].

Proposition 1. Let $\mu \in \mathbb{R}, E$ be a set of class $\mathcal{C}^{1,1}$ and $x \in \partial E$. Assume that there exists an open set $A \ni x$ such that $A \cap \partial E$ is the graph of a $\mathcal{C}^{1,1}$ function, and

$$
P(E, A)-\mu|E \cap A| \leq P(E \cup B, A)-\mu|(E \cup B) \cap A|,
$$

for any bounded measurable set $B$ with $\bar{B} \subset A$. Then $(N-1) \mathbf{H}_{E}(x) \geq \mu$ for $\mathcal{H}^{N-1}$-almost every $x \in A \cap \partial E$. Similarly, if in place of (7) there holds the inequality

$$
P(E, A)-\mu|E \cap A| \leq P(E \backslash B, A)-\mu|(E \backslash B) \cap A|,
$$

then $(N-1) \mathbf{H}_{E}(x) \leq \mu$ for $\mathcal{H}^{N-1}$-almost every $x \in A \cap \partial E$.

\subsection{A generalized Green's formula}

Let $\Omega$ be an open subset of $\mathbb{R}^{N}$. Following [8], let

$$
X_{2}(\Omega):=\left\{z \in L^{\infty}\left(\Omega ; \mathbb{R}^{N}\right): \operatorname{div} z \in L^{2}(\Omega)\right\} .
$$

If $z \in X_{2}(\Omega)$ and $w \in L^{2}(\Omega) \cap B V(\Omega)$ we define the functional $(z, D w): C_{0}^{\infty}(\Omega) \rightarrow \mathbb{R}$ by the formula

$$
<(z, D w), \varphi>:=-\int_{\Omega} w \varphi \operatorname{div} z d x-\int_{\Omega} w z \cdot \nabla \varphi d x .
$$


Then $(z, D w)$ is a Radon measure in $\Omega$,

$$
\int_{\Omega}(z, D w)=\int_{\Omega} z \cdot \nabla w d x \quad \forall w \in L^{2}(\Omega) \cap W^{1,1}(\Omega),
$$

and

$$
\left|\int_{B}(z, D w)\right| \leq \int_{B}|(z, D w)| \leq\|z\|_{\infty} \int_{B}|D w| \quad \forall B \text { Borel set } \subseteq \Omega .
$$

We denote by $\theta(z, D w) \in L_{|D w|}^{\infty}(\Omega)$ the density of $(z, D w)$ with respect to $|D w|$, that is

$$
(z, D w)(B)=\int_{B} \theta(z, D w) d|D w| \quad \forall \text { Borel set } B \subseteq \Omega .
$$

We recall the following result proved in [8].

Theorem 1. Let $\Omega \subset \mathbb{R}^{N}$ be a bounded open set with Lipschitz boundary. Let $u \in B V(\Omega) \cap L^{2}(\Omega)$ and $z \in L^{\infty}\left(\Omega ; \mathbb{R}^{N}\right)$ with $\operatorname{div} z \in L^{2}(\Omega)$. Then there exists a function $\left[z \cdot \nu^{\Omega}\right] \in L^{\infty}(\partial \Omega)$ such that $\left\|\left[z \cdot \nu^{\Omega}\right]\right\|_{L^{\infty}(\partial \Omega)} \leq\|z\|_{L^{\infty}\left(\Omega ; \mathbb{R}^{N}\right)}$, and

$$
\int_{\Omega} u \operatorname{div} z d x+\int_{\Omega} \theta(z, D u) d|D u|=\int_{\partial \Omega}\left[z \cdot \nu^{\Omega}\right] u d \mathcal{H}^{N-1}
$$

When $\Omega=\mathbb{R}^{N}$ we have the following integration by parts formula [8], for $z \in X_{2}\left(\mathbb{R}^{N}\right)$ and $w \in L^{2}\left(\mathbb{R}^{N}\right) \cap B V\left(\mathbb{R}^{N}\right):$

$$
\int_{\mathbb{R}^{N}} w \operatorname{div} z d x+\int_{\mathbb{R}^{N}}(z, D w)=0 .
$$

In particular, if $z \in X_{2}\left(\mathbb{R}^{N}\right)$ and $Q$ is bounded and has finite perimeter in $\mathbb{R}^{N}$, from (9) and (8) it follows

$$
\int_{Q} \operatorname{div} z d x=\int_{\mathbb{R}^{N}}\left(z,-D \chi_{Q}\right)=\int_{\partial^{*} Q} \theta\left(z,-D \chi_{Q}\right) d \mathcal{H}^{N-1}
$$

If additionally, $Q$ is a bounded open set with Lipschitz boundary, then $\theta\left(z,-D \chi_{Q}\right)$ coincides with $\left[z \cdot \nu^{Q}\right]$.

Remark 1. Let $\Omega \subset \mathbb{R}^{2}$ be a bounded Lipschitz open set, and let $z_{\text {inn }} \in L^{\infty}\left(\Omega ; \mathbb{R}^{2}\right)$ with $\operatorname{div} z_{\text {inn }} \in$ $L^{2}(\Omega)$, and $z_{\text {out }} \in L^{\infty}\left(\mathbb{R}^{2} \backslash \bar{\Omega} ; \mathbb{R}^{2}\right)$ with $\operatorname{div} z_{\text {out }} \in L_{\text {loc }}^{2}\left(\mathbb{R}^{2} \backslash \bar{\Omega}\right)$. Assume that

$$
\left[z_{\text {inn }} \cdot \nu^{\Omega}\right](x)=-\left[z_{\text {out }} \cdot \nu^{R^{2} \backslash \bar{\Omega}}\right](x) \quad \text { for } \mathcal{H}^{N-1}-\text { a.e } x \in \partial \Omega .
$$

Then if we define $z:=z_{\text {inn }}$ on $\Omega$ and $z:=z_{\text {out }}$ on $\mathbb{R}^{2} \backslash \bar{\Omega}$, we have $z \in L^{\infty}\left(\mathbb{R}^{2} ; \mathbb{R}^{2}\right)$ and $\operatorname{div} z \in L_{\text {loc }}^{2}\left(\mathbb{R}^{2}\right)$.

\subsection{The subdifferential of the total variation. Calibrable sets}

Consider the energy functional $\Psi: L^{2}\left(\mathbb{R}^{N}\right) \rightarrow(-\infty,+\infty]$ defined by

$$
\Psi(u):=\left\{\begin{array}{ccc}
\int_{\mathbb{R}^{N}}|D u| & \text { if } & u \in L^{2}\left(\mathbb{R}^{N}\right) \cap B V\left(\mathbb{R}^{N}\right) \\
+\infty & \text { if } & u \in L^{2}\left(\mathbb{R}^{N}\right) \backslash B V\left(\mathbb{R}^{N}\right) .
\end{array}\right.
$$

Since the functional $\Psi$ is convex, lower semicontinuous and proper, then $\partial \Psi$ is a maximal monotone operator with dense domain, generating a contraction semigroup in $L^{2}\left(\mathbb{R}^{N}\right)$ (see [15]). Next Lemma gives the characterization of $\partial \Psi$ (see $[5,11]$ for a proof).

Lemma 1. The following assertions are equivalent:

(a) $v \in \partial \Psi(u)$; 
(b)

$$
\begin{gathered}
u \in L^{2}\left(\mathbb{R}^{N}\right) \cap B V\left(\mathbb{R}^{N}\right), v \in L^{2}\left(\mathbb{R}^{N}\right), \\
\exists z \in X_{2}\left(\mathbb{R}^{N}\right) \text { with }\|z\|_{\infty} \leq 1, \text { such that } v=-\operatorname{div} z \text { in } \mathcal{D}^{\prime}\left(\mathbb{R}^{N}\right),
\end{gathered}
$$

and

$$
\int_{\mathbb{R}^{N}}(z, D u)=\int_{\mathbb{R}^{N}}|D u|
$$

From now on we shall write $v=\operatorname{div}\left(\frac{D u}{|D u|}\right)$ instead of $v \in \partial \Psi(u)$.

Given a function $g \in L^{2}\left(\mathbb{R}^{N}\right)$, we define

$$
\|g\|_{*}:=\sup \left\{\left|\int_{\mathbb{R}^{N}} g(x) u(x) d x\right|: u \in L^{2}\left(\mathbb{R}^{N}\right) \cap B V\left(\mathbb{R}^{N}\right), \int_{\mathbb{R}^{N}}|D u| \leq 1\right\} .
$$

Note that $\|g\|_{*}$ may be infinite. Let us recall the following Lemma ([11],[34]).

Lemma 2. Let $f \in L^{2}\left(\mathbb{R}^{N}\right)$ and $\lambda>0$. The following assertions hold.

(a) the function $u$ is the solution of

$$
\min _{w \in L^{2}\left(\mathbb{R}^{N}\right) \cap B V\left(\mathbb{R}^{N}\right)} D(w), \quad D(w):=\int_{\mathbb{R}^{N}}|D w|+\frac{\lambda}{2} \int_{\mathbb{R}^{N}}(w-f)^{2} d x
$$

if and only if there exists $z \in X_{2}\left(\mathbb{R}^{N}\right)$ satisfying (13) with $\|z\|_{\infty} \leq 1$ and $-\lambda^{-1} \operatorname{div} z=f-u$.

(b) The function $u \equiv 0$ is the solution of (14) if and only if $\|f\|_{*} \leq \frac{1}{\lambda}$.

(c) We have $\partial \Psi(0)=\left\{f \in L^{2}\left(\mathbb{R}^{N}\right):\|f\|_{*} \leq 1\right\}$.

Obviously, part $(a)$ follows from Lemma 1 since $\partial \Psi(u)+\lambda(u-f) \ni 0$ is the Euler-Lagrange equation for (14). Part (b) can be found in $([11],[34])$, and it is easily deduced from $(a)$. Part $(c)$ follows from $(a)$ and $(b)$, or as an immediate consequence of duality.

Remark 2. We observe that if $z \in X_{2}\left(\mathbb{R}^{N}\right), u \in B V\left(\mathbb{R}^{N}\right)$, and $(z, D u)=|D u|$ then $\left|\left(z, D \chi_{\{u \geq t\}}\right)\right|=$ $\left|D \chi_{\{u \geq t\}}\right|$ as measures in $\mathbb{R}^{N}$ for almost any $t \in \mathbb{R}$. Indeed, by [8, Proposition 2.7], we have

$$
<(z, D u), \varphi>=\int_{-\infty}^{\infty}<\left(z, D \chi_{\{u \geq t\}}\right), \varphi>d t, \quad \varphi \in C_{0}^{\infty}(\Omega)
$$

Since $|D u|(\varphi)=\int_{-\infty}^{\infty}\left|D \chi_{\{u \geq t\}}\right|(\varphi)$, we may write $(z, D u)=|D u|$ as

$$
\int_{-\infty}^{\infty}<\left(z, D \chi_{\{u \geq t\}}\right), \varphi>d t=\int_{-\infty}^{\infty}\left|D \chi_{\{u \geq t\}}\right|(\varphi) d t, \quad \varphi \in C_{0}^{\infty}\left(\mathbb{R}^{N}\right)
$$

and this implies our claim.

Definition 1. Let $E$ be a bounded set of finite perimeter in $\mathbb{R}^{N}$. We say that $E$ is calibrable if there exists a vector field $\xi \in L^{\infty}\left(\mathbb{R}^{N}, \mathbb{R}^{N}\right)$ with $\|\xi\|_{\infty} \leq 1$ such that $\left(\xi, D \chi_{E}\right)=\left|D \chi_{E}\right|$ as measures in $R^{N}$, and

$$
-\operatorname{div} \xi=\lambda_{E} \chi_{E} \quad \text { in } \mathcal{D}^{\prime}\left(\mathbb{R}^{N}\right)
$$

for some constant $\lambda_{E}$. 
Notice that, a set of finite perimeter $E$ is calibrable if and only if it exists $\lambda_{E} \in \mathbb{R}$ such that $\lambda_{E} \chi_{E} \in \partial \Psi\left(\chi_{E}\right)$. Observe that if $E$ is calibrable, then $\lambda_{E}=\frac{P(E)}{|E|}$. Indeed, multiplying (15) by $\chi_{E}$ and integrating in $\mathbb{R}^{N}$ we obtain

$$
\lambda_{E}|E|=-\int_{\mathbb{R}^{N}} \operatorname{div} \xi \chi_{E} d x=\int_{\mathbb{R}^{N}}\left(\xi, D \chi_{E}\right)=\int_{\mathbb{R}^{N}}\left|D \chi_{E}\right|=P(E) .
$$

Let us recall the following result (see, for instance, [11]). We shall include its proof for the sake of completeness.

Proposition 2. Let $E$ be a bounded set of finite perimeter in $\mathbb{R}^{N}$. Assume $E$ to be convex. The following assertions are equivalent

(i) E is calibrable

(ii) E minimizes the functional

$$
P(X)-\lambda_{E}|X|
$$

on the sets of finite perimeter $X \subseteq E$.

Proof: $(i) \rightarrow($ ii $)$ Let $X$ be a set of finite perimeter in $\mathbb{R}^{N}$. We have

$$
\lambda_{E}|E \cap X|=-\int_{\mathbb{R}^{N}} \operatorname{div} \xi \chi_{X} d x=\int_{\mathbb{R}^{N}}\left(\xi, D \chi_{X}\right) \leq P(X) .
$$

Hence $P(X)-\lambda_{E}|X| \geq 0=P(E)-\lambda_{E}|E|$ for any set of finite perimeter $X \subseteq E$.

$($ ii $) \rightarrow($ i $)$ Let us prove that the function $f:=\lambda_{E} \chi_{E}$ satisfies $\|f\|_{*} \leq 1$. Indeed, if $w \in L^{2}\left(\mathbb{R}^{N}\right) \cap$ $B V\left(\mathbb{R}^{N}\right)$ is nonnegative, we have

$$
\begin{aligned}
\int_{\mathbb{R}^{N}} f(x) w(x) d x & =\int_{0}^{\infty} \int_{\mathbb{R}^{N}} \lambda_{E} \chi_{E} \chi_{\{w \geq t\}} d x d t=\int_{0}^{\infty} \lambda_{E}|E \cap\{w \geq t\}| d t \\
& \leq \int_{0}^{\infty} P(E \cap\{w \geq t\}) d t \leq \int_{0}^{\infty} P(\{w \geq t\}) d t=\int_{\mathbb{R}^{N}}|D w|
\end{aligned}
$$

where we have used that for all $t \geq 0$ for which $\{w \geq t\}$ is a set of finite perimeter we have that

$$
P(E \cap\{w \geq t\}) \leq P(\{w \geq t\})
$$

which is a consequence of the convexity of $E$ (see, for instance, [3]). Splitting any function $\omega \in$ $L^{2}\left(\mathbb{R}^{N}\right) \cap B V\left(\mathbb{R}^{N}\right)$ into its positive and negative part, using the above inequality one can prove that $\left|\int_{\mathbb{R}^{N}} f(x) \omega(x) d x\right| \leq \int_{\mathbb{R}^{N}}|D \omega|$. It follows that $\|f\|_{*} \leq 1$. Then, by Lemma 2 , there is a vector field $\xi \in L^{\infty}\left(\mathbb{R}^{N} ; \mathbb{R}^{N}\right)$ with $\|\xi\|_{\infty} \leq 1$ such that

$$
-\operatorname{div} \xi=f=\lambda_{E} \chi_{E} .
$$

Now, multiplying (17) by $\chi_{C}$ and integrating by parts, we obtain

$$
\int_{\mathbb{R}^{N}}\left(\xi, D \chi_{E}\right)=\lambda_{E} \int_{\mathbb{R}^{2}} \chi_{E} d x=P(E)=\int_{\mathbb{R}^{N}}\left|D \chi_{E}\right|,
$$

hence $\chi_{E}$ is calibrable.

Proposition 3. Let $B$ be a ball in $\mathbb{R}^{N}$, and $\lambda>0$. The solution of

$$
u-\lambda^{-1} \operatorname{div}\left(\frac{D u}{|D u|}\right)=\chi_{B}
$$

is $u=\left(1-\frac{\lambda_{B}}{\lambda}\right)^{+} \chi_{B}$.

For a proof, we refer to [11], [5]. 


\subsection{The minimizing TV flow}

The following notion of strong solution is adapted from the notion of strong solution in semigroup sense [15] (see also [5], [11]).

Definition 2. A function $u \in C\left([0, T] ; L^{2}\left(\mathbb{R}^{N}\right)\right)$ is called a strong solution of (2) if

$$
u \in W_{\mathrm{loc}}^{1,2}\left(0, T ; L^{2}\left(\mathbb{R}^{N}\right)\right) \cap L_{w}^{1}(] 0, T\left[; B V\left(\mathbb{R}^{N}\right)\right)
$$

and there exists $z \in L^{\infty}(] 0, T\left[\times \mathbb{R}^{N} ; \mathbb{R}^{N}\right)$ with $\|z\|_{\infty} \leq 1$ such that

$$
u_{t}=\operatorname{div} z \quad \text { in } \mathcal{D}^{\prime}(] 0, T\left[\times \mathbb{R}^{N}\right)
$$

and

$$
\int_{\mathbb{R}^{N}}(z(t), D u(t))=\int_{\mathbb{R}^{N}}|D u(t)| \quad t>0 \text { a.e.. }
$$

Theorem 2. Let $u_{0} \in L^{2}\left(\mathbb{R}^{N}\right)$. Then there exists a unique strong solution in the semigroup sense $u$ of (2) in $[0, T]$ for every $T>0$, i.e., $u \in C\left([0, T] ; L^{2}\left(\mathbb{R}^{N}\right)\right) \cap W_{\mathrm{loc}}^{1,2}\left(0, T ; L^{2}\left(\mathbb{R}^{N}\right)\right), u(t) \in D(\partial \Phi)$ a.e. in $t \in[0, T]$ and

$$
-u^{\prime}(t) \in \partial \Phi(u(t)) \quad \text { a.e. in } t \in[0, T] .
$$

Moreover, any semigroup solution is a strong solution, and conversely. Finally, if $u$ and $v$ are the strong solutions of (2) corresponding to the initial conditions $u_{0}, v_{0} \in L^{2}(\Omega)$, then

$$
\|u(t)-v(t)\|_{2} \leq\left\|u_{0}-v_{0}\right\|_{2} \text { for any } t>0 .
$$

\subsection{The Dirichlet problem}

Let $\Omega$ be an open bounded subset of $\mathbb{R}^{N}$ with Lipschitz boundary, and $\varphi \in L^{1}(\Omega)$. Let $\Psi_{\varphi}: L^{2}(\Omega) \rightarrow$ $(-\infty,+\infty]$ be the functional defined by

$$
\Psi_{\varphi}(u):=\left\{\begin{array}{ccc}
\int_{\Omega}|D u|+\int_{\partial \Omega}|u-\varphi| & \text { if } & u \in L^{2}(\Omega) \cap B V(\Omega) \\
+\infty & \text { if } & u \in L^{2}(\Omega) \backslash B V(\Omega) .
\end{array}\right.
$$

The functional $\Psi_{\varphi}$ is convex and lower semicontinuous in $L^{2}(\Omega)$, hence $\partial \Psi_{\varphi}$ is a maximal monotone operator in $L^{2}(\Omega)$.

Let us recall the characterization of $\partial \Psi_{\varphi}$ given in [6].

Theorem 3. The following conditions are equivalent

(i) $v \in \partial \Psi_{\varphi}(u)$

(ii) $u, v \in L^{2}(\Omega), u \in B V(\Omega)$ and there exists $z \in X(\Omega)$ with $\|z\|_{\infty} \leq 1, v=-\operatorname{div}(z)$ in $\mathcal{D}^{\prime}(\Omega)$ such that

$$
\int_{\Omega}(w-u) v \leq \int_{\Omega} z \cdot \nabla w-\|D u\|+\int_{\partial \Omega}|w-\varphi|-\int_{\partial \Omega}|u-\varphi|,
$$

$\forall w \in W^{1,1}(\Omega) \cap L^{\infty}(\Omega)$.

(iii) $u, v \in L^{2}(\Omega), u \in B V(\Omega)$ and there exists $z \in X(\Omega)$ with $\|z\|_{\infty} \leq 1, v=-\operatorname{div}(z)$ in $\mathcal{D}^{\prime}(\Omega)$ such that $(z, D u)=|D u|$ and $\left[z \cdot \nu^{\Omega}\right] \in \operatorname{sign}(\varphi-u) \mathcal{H}^{N-1}$ a.e. on $\partial \Omega$.

The following result was proved in [6]. 
Theorem 4. Let $f_{i} \in L^{2}(\Omega), \varphi_{i} \in L^{1}(\partial \Omega), i=1,2$. Assume that $f_{1} \leq f_{2}$ and $\varphi_{1} \leq \varphi_{2}$. Let $u_{i}$, $i=1,2$, be the solution of

$$
u+\lambda \partial \Psi_{\varphi_{i}}(u) \ni f_{i}
$$

then $u_{1} \leq u_{2}$.

Let $\epsilon>0$.

$$
\Psi_{\varphi}^{\epsilon}(u):=\left\{\begin{array}{cl}
\int_{\Omega} \sqrt{\epsilon^{2}+|D u|}+\int_{\partial \Omega}|u-\varphi| & \text { if } \quad u \in L^{2}(\Omega) \cap B V(\Omega) \\
+\infty & \text { if } \quad u \in L^{2}(\Omega) \backslash B V(\Omega) .
\end{array}\right.
$$

By the results in [32],[6], we know that $\partial \Psi_{\varphi}^{\epsilon}$ is a maximal monotone operator which can be characterized in an analogous way that $\partial \Psi_{\varphi}$, and Theorem 4 also holds for $\partial \Psi_{\varphi}^{\epsilon}$. Moreover, as $\epsilon \rightarrow 0$, the solutions of

$$
u+\lambda \partial \Psi_{\varphi}^{\epsilon}(u) \ni f
$$

where $f \in L^{2}(\Omega)$ converge to the solution of $u+\lambda \partial \Psi_{\varphi}(u) \ni f$.

\section{A characterization of convex calibrable sets}

\subsection{Properties of level sets of the solution of a Variational Problem}

Proposition 4. Let $C$ be a bounded convex subset of $\mathbb{R}^{N}$. Let $u \in B V\left(\mathbb{R}^{N}\right) \cap L^{2}\left(\mathbb{R}^{N}\right)$ be the solution of the variational problem

$$
(Q)_{\lambda}: \quad \min _{u \in B V\left(\mathbb{R}^{N}\right) \cap L^{2}\left(\mathbb{R}^{N}\right)}\left\{\int_{\mathbb{R}^{N}}|D u|+\frac{\lambda}{2} \int_{\mathbb{R}^{N}}\left(u-\chi_{C}\right)^{2} d x\right\} .
$$

Then $0 \leq u \leq 1$. Let $E_{s}:=[u \geq s], s \in(0,1]$. Then $E_{s} \subseteq C$, and, for any $s \in(0,1]$, we have

$$
P\left(E_{s}\right)-\lambda(1-s)\left|E_{s}\right| \leq P(F)-\lambda(1-s)|F|
$$

for any $F \subseteq C$.

Proof. Recall that $u$ satisfies the following partial differential equation

$$
u-\lambda^{-1} \operatorname{div}\left(\frac{D u}{|D u|}\right)=\chi_{C} \quad \text { in } \mathbb{R}^{N} .
$$

Let $u^{-}=\min (u, 0)$. Multiplying $(26)$ by $u^{-}$and integrating by parts, we deduce that $u^{-}=0$. Similarly, multiplying $(26)$ by $(u-1)^{+}$we deduce that $u \leq 1$. Let us prove that $u=0$ outside $C$. Let $H$ be a half-plane containing $C$. Since $\chi_{C} \leq \chi_{H}$, and $v=\chi_{H}$ is the solution of (26) with right-hand side equal to $v$, by the comparison principle proved in [11] we have that $u \leq \chi_{H}$. This implies that $u=0$ outside $C$. This implies that $E_{s} \subseteq C$ for all $s \in(0,1]$.

Let $F \subseteq C$ be a set of finite perimeter. By Remark 2, we have that $\left(z, D \chi_{E_{s}}\right)=\left|D \chi_{E_{s}}\right|$ for almost all $s \in(0,1]$. Hence, for such an $s \in(0,1]$, we have

$-\int_{\mathbb{R}^{N}} \operatorname{div} z\left(\chi_{F}-\chi_{E_{s}}\right) d x=\int_{\mathbb{R}^{N}}\left(z, D \chi_{F}\right)-\int_{\mathbb{R}^{N}}\left(z, D \chi_{E_{s}}\right)=\int_{\mathbb{R}^{N}}\left(z, D \chi_{F}\right)-P\left(E_{s}\right) \leq P(F)-P\left(E_{s}\right)$

and we deduce

$$
P(F)-P\left(E_{s}\right) \geq \lambda \int_{\mathbb{R}^{N}}\left(\chi_{C}-u\right)\left(\chi_{F}-\chi_{E_{s}}\right)=\lambda \int_{\mathbb{R}^{N}}\left(\left(\chi_{C}-s\right)+(s-u)\right)\left(\chi_{F}-\chi_{E_{s}}\right) .
$$


Since $(s-u)\left(\chi_{F}-\chi_{E_{s}}\right) \geq 0$ we have

$$
P(F)-P\left(E_{s}\right) \geq \lambda \int_{\mathbb{R}^{N}}\left(\chi_{C}-s\right)\left(\chi_{F}-\chi_{E_{s}}\right)=\lambda(1-s)\left(|F|-\left|E_{s}\right|\right) .
$$

Since all sets $E_{s}$ are contained in $C$, the perimeter is lower semicontinuous, and the area is continuous for increasing or decreasing families of sets contained in $C$, we deduce that (25) holds for any $s \in$ $(0,1]$.

Lemma 3. Let $C$ be a bounded convex subset of $\mathbb{R}^{N}$ of positive measure. Let $u_{\lambda}$ be the solution of $(Q)_{\lambda}, \lambda>0$.

(i) $u_{\lambda} \neq \chi_{C}$ for any $\lambda>0$.

(ii) $u_{\lambda} \rightarrow \chi_{C}$ in $L^{2}\left(\mathbb{R}^{N}\right)$ as $\lambda \rightarrow \infty$.

(iii) Assume that $C$ has bounded mean curvature. Let $\Lambda:=(N-1)\left\|\mathbf{H}_{C}\right\|_{\infty}$. For any $\lambda>0$, we have

$$
u_{\lambda} \geq\left(1-N \frac{\Lambda}{\lambda}\right)^{+} \chi_{C}
$$

(iv) $u_{\lambda} \neq 0$ if and only if $\lambda>\frac{1}{\left\|\chi_{C}\right\|_{B V^{*}}}$.

(v) Assume that $C$ is not calibrable (i.e., it does not exist a vector field $z \in L^{\infty}\left(\mathbb{R}^{N}, \mathbb{R}^{N}\right),\|z\|_{\infty} \leq 1$ such that - div $\left.z=\lambda_{C} \chi_{C}\right)$. For any $\lambda>\frac{1}{\left\|\chi_{C}\right\|_{B V^{*}}} u_{\lambda}$ cannot be a multiple of $\chi_{C}$. Thus, for any such $\lambda$, there is some $s \in[0,1]$ such that $\left[u_{\lambda} \geq s\right] \neq C$.

Proof: $(i)$ Suppose that there is $\lambda>0$ such that $u_{\lambda}=\chi_{C}$. Then there is a vector field $z_{\lambda} \in$ $L^{\infty}\left(\mathbb{R}^{N}, \mathbb{R}^{N}\right),\left\|z_{\lambda}\right\|_{\infty} \leq 1$, such that $\left(z_{\lambda}, D \chi_{C}\right)=\left|D \chi_{C}\right|$ and

$$
\operatorname{div} z_{\lambda}=0 .
$$

Multiplying this equation by $\chi_{C}$ and integrating in $\mathbb{R}^{N}$, we obtain

$$
0=-\int_{\mathbb{R}^{N}} \operatorname{div} z_{\lambda} \chi_{C} d x=\int_{\mathbb{R}^{N}}\left(z_{\lambda}, D \chi_{C}\right)=\int_{\mathbb{R}^{N}}\left|D \chi_{C}\right|=P(C) .
$$

This contradiction proves that $u_{\lambda} \neq \chi_{C}$.

(ii) Since

$$
\int_{\mathbb{R}^{N}}\left|D u_{\lambda}\right|+\frac{\lambda}{2} \int_{\mathbb{R}^{N}}\left(u_{\lambda}-\chi_{C}\right)^{2} d x \leq \int_{\mathbb{R}^{N}}\left|D \chi_{C}\right|=P(C)
$$

we deduce that

$$
\int_{\mathbb{R}^{N}}\left(u_{\lambda}-\chi_{C}\right)^{2} d x \leq \frac{2}{\lambda} P(C)
$$

i.e. $u_{\lambda} \rightarrow \chi_{C}$ in $L^{2}$ as $\lambda \rightarrow \infty$. Moreover, $u_{\lambda}$ is bounded in $B V\left(\mathbb{R}^{N}\right)$.

(iii) By definition of $\Lambda$, we have that each principal curvature of $\partial C$ is $\leq \Lambda$. Thus there is a ball $B$ of radius $\frac{1}{\Lambda}$ which is osculating at each point of $\partial C$ from inside ([39], Corollary 3.2.10). Let $p \in \partial C$, let $B_{p}$ be the corresponding osculating ball. Let us compare $u_{\lambda}$ with the solution $u_{p}$ of

$$
u-\lambda^{-1} \operatorname{div}\left(\frac{D u}{|D u|}\right)=\chi_{B_{p}}
$$

Since $\chi_{B_{p}} \leq \chi_{C}$, by the comparison principle [11] we deduce that $u_{p} \leq u_{\lambda}$. The solution $u_{p}$ is given explicitly by

$$
u_{p}=\left(1-\frac{\lambda_{B_{p}}}{\lambda}\right)^{+} \chi_{B_{p}}
$$


But

$$
\lambda_{B_{p}}=\frac{P\left(B_{p}\right)}{\left|B_{p}\right|}=\frac{N}{1 / \Lambda}=N \Lambda
$$

Hence

$$
u_{p}=\left(1-N \frac{\Lambda}{\lambda}\right)^{+} \chi_{B_{p}} .
$$

Since this is true for any $p \in \partial C$, and also for any $p$ in the interior of $C$, we deduce that

$$
u_{\lambda} \geq\left(1-N \frac{\Lambda}{\lambda}\right)^{+} \chi_{C}
$$

(iv) We know that $u_{\lambda}$ is characterized by the solution of

$$
u_{\lambda}-\lambda^{-1} \operatorname{div} z_{\lambda}=\chi_{C}
$$

where $z_{\lambda} \in L^{\infty}\left(\mathbb{R}^{N}, \mathbb{R}^{N}\right),\left\|z_{\lambda}\right\|_{\infty} \leq 1$, with $\left(z_{\lambda}, D u_{\lambda}\right)=\left|D u_{\lambda}\right|$. Thus $u_{\lambda}=0$ if and only if - div $z_{\lambda}=$ $\lambda \chi_{C}$, i.e., if and only if $\left\|\lambda \chi_{C}\right\|_{B V^{*}} \leq 1$. Statement (iv) is proved.

(v) Suppose that for some $\lambda>\frac{1}{\left\|\chi_{C}\right\|_{B V^{*}}}$, we have $u_{\lambda}=c_{\lambda} \chi_{C}$ for some constant $0 \leq c_{\lambda} \leq 1$. Observe that, by $(i), c_{\lambda}<1$, and, by $(i v), c_{\lambda}>0$. Then

$$
-\operatorname{div} z_{\lambda}=\lambda\left(1-c_{\lambda}\right) \chi_{C}
$$

Since $\left(z_{\lambda}, D u_{\lambda}\right)=\left|D u_{\lambda}\right|$, and $c_{\lambda}>0$, we have that $\left(z_{\lambda}, D \chi_{C}\right)=\left|D \chi_{C}\right|=P(C)$. Multiplying the PDE by $\chi_{C}$ and integrating by parts we deduce that

$$
\lambda\left(1-c_{\lambda}\right)=\lambda_{C}
$$

Hence

$$
-\operatorname{div} z_{\lambda}=\lambda_{C} \chi_{C},
$$

and therefore $C$ is calibrable, a contradiction. The final assertion is a simple consequence of the first.

Lemma 4. For any $\lambda>0$, let us consider the problem

$$
(P)_{\lambda}: \quad \min _{F \subseteq C} P(F)-\lambda|F| .
$$

Then

(i) Let $C_{\lambda}, C_{\mu}$ be minimizers of $(P)_{\lambda}$, and $(P)_{\mu}$ respectively. If $\lambda<\mu$, then $C_{\lambda} \subseteq C_{\mu}$.

(ii) Let $\mu>\lambda$. Assume that $C$ is a solution of $(P)_{\lambda}$. Then $C$ is a solution of $(P)_{\mu}$.

(iii) Let $\lambda_{n} \uparrow \lambda$. Then $C_{\lambda}^{\cup}:=\cup_{n} C_{\lambda_{n}}$ is a minimizer of $(P)_{\lambda}$. Moreover $P\left(C_{\lambda_{n}}\right) \rightarrow P\left(C_{\lambda}^{\cup}\right)$. Similarly, if $\lambda_{n} \downarrow \lambda$, then $C_{\lambda}^{\cap}:=\cap_{n} C_{\lambda_{n}}$ is a minimizer of $(P)_{\lambda}$, and $P\left(C_{\lambda_{n}}\right) \rightarrow P\left(C_{\lambda}^{\cap}\right)$.

(iv) Assume that $C$ has bounded mean curvature. Let $\Lambda:=(N-1)\left\|\mathbf{H}_{C}\right\|_{\infty}$. Then $C$ is a solution of $(P)_{\lambda}$ for any $\lambda \geq N \Lambda$.

Proof: $(i)$ Observe that we have

$$
\begin{aligned}
& P\left(C_{\lambda}\right)-\lambda\left|C_{\lambda}\right| \leq P\left(C_{\lambda} \cap C_{\mu}\right)-\lambda\left|C_{\lambda} \cap C_{\mu}\right| \\
& P\left(C_{\mu}\right)-\mu\left|C_{\mu}\right| \leq P\left(C_{\lambda} \cup C_{\mu}\right)-\mu\left|C_{\lambda} \cup C_{\mu}\right| .
\end{aligned}
$$

Adding both inequalities and using that for any two sets of finite perimeter $X, Y$ in $\mathbb{R}^{N}$ we have

$$
P(X \cap Y)+P(X \cup Y) \leq P(X)+P(Y)
$$


we obtain that

$$
\lambda\left(\left|C_{\lambda} \cap C_{\mu}\right|-\left|C_{\lambda}\right|\right) \leq \mu\left(\left|C_{\mu}\right|-\left|C_{\lambda} \cup C_{\mu}\right|\right)
$$

i.e.,

$$
\mu\left|C_{\lambda} \backslash C_{\mu}\right| \leq \lambda\left|C_{\lambda} \backslash C_{\mu}\right| .
$$

Since $\lambda<\mu$, this implies that $\left|C_{\lambda} \backslash C_{\mu}\right|=0$, hence $C_{\lambda} \subseteq C_{\mu}$.

(ii) It follows as a consequence of $(i)$.

(iii) Let $\lambda_{n} \uparrow \lambda$. For each $n$ and each $F \subseteq C$, we have

$$
P\left(C_{\lambda_{n}}\right)-\lambda_{n}\left|C_{\lambda_{n}}\right| \leq P(F)-\lambda_{n}|F|
$$

Using the lower semicontinuity of the perimeter we deduce that

$$
P\left(C_{\lambda}^{\cup}\right)-\lambda\left|C_{\lambda}^{\cup}\right| \leq P(F)-\lambda|F|,
$$

i.e, $C_{\lambda}^{\cup}$ is a minimizer of $(P)_{\lambda}$. Now, taking limsup in

$$
P\left(C_{\lambda_{n}}\right)-\lambda_{n}\left|C_{\lambda_{n}}\right| \leq P\left(C_{\lambda}^{\cup}\right)-\lambda_{n}\left|C_{\lambda}^{\cup}\right|
$$

we have that

$$
\limsup _{n} P\left(C_{\lambda_{n}}\right) \leq P\left(C_{\lambda}^{\cup}\right) .
$$

Using this, together with the lower semicontinuity of the perimeter, we deduce that $\lim _{n} P\left(C_{\lambda_{n}}\right)=$ $P\left(C_{\lambda}^{\cup}\right)$. The proof of the other assertion is similar.

(iv) By (ii), it suffices to prove that $C$ is a solution of $(P)_{N \Lambda}$. For that let $\eta>N \Lambda$. Take $0<$ $s_{n}<1-N \frac{\Lambda}{\eta}$ such that $\eta\left(1-s_{n}\right) \downarrow N \Lambda$. We observe that, by Lemma 3.(iii), $\left[u_{\eta} \geq s_{n}\right]=C$ and, by Proposition 4 , is a minimum of

$$
P(F)-\eta\left(1-s_{n}\right)|F| .
$$

Now, by assertion (iii) in the present Lemma, we deduce that $C$ is also a minimum of

$$
P(F)-N \Lambda|F|
$$

Remark 3. In Proposition 4 we have proved that for any $s \in(0,1]$, the level set $\left[u_{\lambda} \geq s\right]$ is a minimizer of $(P)_{\lambda(1-s)}$. Moreover, by Lemma 4, the sets $\left[u_{\lambda} \geq s\right]^{\cup}:=\cup_{\epsilon>0}\left[u_{\lambda} \geq s+\epsilon\right], s \in[0,1)$, and $\left[u_{\lambda} \geq s\right]^{\cap}:=\cap_{\epsilon>0}\left[u_{\lambda} \geq s-\epsilon\right], s \in(0,1]$, are also minimizers of $(P)_{\lambda(1-s)}$ (obviously $\left[u_{\lambda} \geq 1\right]^{\cup}=\emptyset$ is also a minimizer of $\left.(P)_{0}\right)$. Notice that, except on countably many values of $s$, they coincide with $\left[u_{\lambda} \geq s\right]$.

\subsection{The concavity of solutions of $(Q)_{\lambda}$}

Our purpose is to prove the following result.

Theorem 5. Let $C$ be a bounded convex domain in $\mathbb{R}^{N}$ of class $C^{1,1}$ such that $(N-1) \mathbf{H}_{C} \leq \frac{1}{R}$, $R>0$. If $\lambda \geq \frac{2 N}{R}$, then the solution $u_{\lambda}$ of $(Q)_{\lambda}$ is concave in $C$. In particular $\left[u_{\lambda} \geq s\right]$ is convex for any $s \in[0,1]$.

Before going into the proof, we observe that, being concave in $C, u_{\lambda}$ is continuous in $C$. In particular $\left[u_{\lambda} \geq s\right]^{\cap}=\left[u_{\lambda} \geq s\right]$ and $\left[u_{\lambda} \geq s\right]^{\cup}=\left[u_{\lambda}>s\right]$. Moreover $\left[u_{\lambda} \geq s\right]=\left[u_{\lambda}>s\right]$ (modulo a null set) for any $s \in\left(0, \max \left(u_{\lambda}\right)\right)$.

For that we recall two auxiliary results. The following theorem was proved by Korevaar in [30] 
Theorem 6. Let $\Omega$ be a $C^{1}$, strictly convex bounded domain in $\mathbb{R}^{N}$. Let $b: \mathbb{R} \times \mathbb{R}^{N} \rightarrow \mathbb{R}$ be such that

$$
\frac{\partial b}{\partial u}>0 \quad \frac{\partial^{2} b}{\partial u^{2}} \geq 0
$$

Assume that $u \in C(\bar{\Omega}) \cap C^{2}(\Omega)$ satisfies

$$
\operatorname{div}\left(\frac{D u}{\sqrt{1+|D u|^{2}}}\right)=b(u, D u)
$$

and the graph of $u$ is a $C^{1}$ surface above $\bar{\Omega}$ making zero contact angle with $\partial \Omega \times \mathbb{R}$, i.e.,

$$
\frac{D u}{\sqrt{1+|D u|^{2}}} \cdot \nu^{\Omega}=-1
$$

Then $u$ is a concave function.

The sense of the boundary condition (31) will be made precise during the proof of Theorem 5 . Let us recall the following result which was proved by Atkinson and Peletier in [9].

Theorem 7. Let $f:\left[\gamma_{0}, \gamma\right] \rightarrow \mathbb{R}$ be a Lipschitz function such that $f>0$ on $\left[\gamma_{0}, \gamma\right]$. Let us consider the problem

$$
\operatorname{div}\left(\frac{D u}{\sqrt{1+|D u|^{2}}}\right)+f(u)=0 \quad \text { in } \quad \mathbb{R}^{N}, N \geq 2 .
$$

Let

$$
f_{m}=\min _{u \in\left[\gamma_{0}, \gamma\right]} f(u), \quad f_{M}=\max _{u \in\left[\gamma_{0}, \gamma\right]} f(u)
$$

and let $L=\frac{N-1}{N}$. Assume that

$$
\left(\gamma-\gamma_{0}\right)\left(f_{m}-L f_{M}\right) \geq 1 .
$$

Then there are numbers $\tilde{R}>0$ and $U \in\left(\gamma_{0}, \gamma\right)$ and a radial solution of (32) such that

$$
\begin{gathered}
0>u^{\prime}(r)>-\infty, \quad \gamma_{0}<u(r)<\gamma \quad \text { for } 0<r<\tilde{R}, \text { and } \\
u^{\prime}(r) \rightarrow-\infty, \quad u(r) \rightarrow U \quad \text { as } r \rightarrow \tilde{R}-.
\end{gathered}
$$

and satisfying the inequalities

$$
\begin{gathered}
\frac{1}{f_{M}-L f_{m}} \leq \tilde{R} \leq \frac{1}{f_{m}-L f_{M}} \\
\gamma-\frac{1}{f_{m}-L f_{M}} \leq U \leq \gamma-\frac{1}{f_{M}-L f_{m}}
\end{gathered}
$$

Theorem 8. Let $C$ be a bounded convex domain in $\mathbb{R}^{N}$ of class $C^{1,1}$. Assume that $(N-1) \mathbf{H}_{C} \leq \frac{1}{R}$, $R>0$. Let $\lambda \geq \frac{2 N}{R}$. Let us consider the following problem

$$
\begin{array}{ll}
u-\lambda^{-1} \operatorname{div}\left(\frac{D u}{\sqrt{\epsilon^{2}+|D u|^{2}}}\right)=1 & \text { in } \quad C \\
u=0 & \text { on } \quad \partial C
\end{array}
$$

Then there is a unique solution $u^{\epsilon}$ of (36) such that $0 \leq u^{\epsilon} \leq 1$. Moreover $u^{\epsilon} \geq \alpha>0$ in a neighborhood of $\partial C$ for some $\alpha>0$. Hence, $u$ satisfies

$$
\left[\frac{D u^{\epsilon}}{\sqrt{\epsilon^{2}+\left|D u^{\epsilon}\right|^{2}}} \cdot \nu^{C}\right]=\operatorname{sign}\left(0-u^{\epsilon}\right)=-1 \quad \text { on } \partial C .
$$


Proof. Existence and uniqueness of a solution $u^{\epsilon}$ of (36) satisfying the Dirichlet boundary condition in the generalized sense follows by the results in [7],[32]. Multiplying (36) by test functions as in the proof of Proposition 4 and integrating by parts we deduce that $0 \leq u^{\epsilon} \leq 1$.

Let us prove that $u^{\epsilon} \geq \alpha>0$ for some $\alpha>0$. For that we shall use Theorem 7 . Since $(N-1) \mathbf{H}_{C} \leq$ $\frac{1}{R}$, by [39] Corollary 3.2.10, we know that at each point $p \in \partial C$, there is a ball $B_{p}$ of radius $R$ such that $B_{p} \subseteq C$ and $p \in \partial B_{p}$.

Lemma 5. There is a radius $\tilde{R} \leq R$ and radial solution $u_{\tilde{B}}$ of

$$
\begin{array}{ll}
u-\lambda^{-1} \operatorname{div}\left(\frac{D u}{\sqrt{\epsilon^{2}+|D u|^{2}}}\right)=1 & \text { in } \quad \tilde{B}=B(0, \tilde{R}) \\
u=0 & \text { on } \quad \partial \tilde{B}
\end{array}
$$

such that

$$
\begin{gathered}
0>u_{\tilde{B}}^{\prime}(r)>-\infty, \quad U<u_{\tilde{B}}(r)<\gamma \quad \text { for } 0<r<\tilde{R}, \text { and } \\
u_{\tilde{B}}^{\prime}(r) \rightarrow-\infty, \quad u_{\tilde{B}}(r) \rightarrow U \quad \text { as } r \rightarrow \tilde{R}-.
\end{gathered}
$$

for some values $\gamma>0, U>0$.

Proof. By rescaling $v_{\tilde{B}}^{\epsilon}(x):=u_{\tilde{B}}\left(\frac{x}{\epsilon}\right)$, we may look for a radial solution of

$$
\begin{array}{ll}
\operatorname{div}\left(\frac{D v}{\sqrt{1+|D v|^{2}}}\right)+\frac{\lambda}{\epsilon}(1-v)=0 & \text { in } \epsilon \tilde{B} \\
v=0 & \text { on } \partial(\epsilon \tilde{B}) .
\end{array}
$$

We shall obtain it as an application of Theorem 7. Let $\gamma_{0}=0$, and $0<\gamma<1$ to be precised in a moment. In this case $f(u)=\frac{\lambda}{\epsilon}(1-u)$, hence

$$
f_{m}=\frac{\lambda}{\epsilon}(1-\gamma), \quad f_{M}=\frac{\lambda}{\epsilon}
$$

and assumption (33) amounts to write

$$
\gamma \frac{\lambda}{\epsilon}(1-N \gamma) \geq N
$$

By Theorem 7 there exists a radius $R_{\lambda}$ satisfying the statement of that Theorem. The inequality (34) can be written as

$$
\frac{N}{1+(N-1) \gamma} \leq \frac{\lambda R_{\lambda}}{\epsilon} \leq \frac{N}{1-N \gamma} \text {. }
$$

Let us fix $\gamma=\frac{1}{2 N}$ and we look for solutions $v$ of (39) bounded by $\gamma=\frac{1}{2 N}$. Assumption (40) can be written as

$$
\lambda \geq 4 N^{2} \epsilon,
$$

and inequality (41) as

$$
\frac{2 N^{2}}{3 N-1} \leq \frac{\lambda R_{\lambda}}{\epsilon} \leq 2 N
$$

We fix $\lambda \geq 4 N^{2} \epsilon$. Since we need that $R_{\lambda} \leq \epsilon R$, by (43), this will be guaranteed if

$$
\frac{2 N \epsilon}{\lambda} \leq \epsilon R
$$

i.e. if $\lambda \geq \frac{2 N}{R}$. Finally, observe that (35) can be written as

$$
\frac{1}{2 N}-\frac{2 N \epsilon}{\lambda} \leq U \leq \frac{1}{2 N}-\frac{2 N^{2} \epsilon}{\lambda(3 N-1)}
$$


Hence, if we take

$$
\lambda>4 N^{2} \epsilon,
$$

then $U=\min v_{\tilde{B}}^{\epsilon}>0$. Summarizing, if we take

$$
\epsilon<\frac{1}{2 N R} \text { and } \quad \lambda \geq \frac{2 N}{R}
$$

then (45) holds and, by Theorem 7, we have a solution $v_{\tilde{B}}^{\epsilon}$ of the PDE in (39) on $\epsilon \tilde{B}$, where $\tilde{B}=B(0, \tilde{R})$ and $\tilde{R}=\frac{R_{\lambda}}{\epsilon} \leq R$, such that $v_{\tilde{B}}^{\epsilon}>0$ on $\partial(\epsilon \tilde{B})$, the graph of $v_{\tilde{B}}^{\epsilon}$ having zero contact angle with $\partial(\epsilon \tilde{B}) \times \mathbb{R}$. Hence there is a solution $u_{\tilde{B}}$ of the PDE in (38) such that $u_{\tilde{B}} \geq \alpha>0$ on $\partial \tilde{B}$, and this solution has zero contact angle with $\partial \tilde{B} \times \mathbb{R}$.

Now, since $u_{\tilde{B}}$ has a zero contact angle with $\partial \tilde{B} \times \mathbb{R}$, we have

$$
\frac{D u_{\tilde{B}}}{\sqrt{\epsilon^{2}+\left|D u_{\tilde{B}}\right|^{2}}} \cdot \nu^{\tilde{B}}=-1=\operatorname{sign}\left(0-u_{\tilde{B}}\right) \quad \text { on } \partial \tilde{B} \text {. }
$$

Since Theorem 3 in Subsection 2.5 also holds for $\partial \Psi_{\varphi}^{\epsilon}[7],[32]$, we deduce that $u_{\tilde{B}}$ represents a solution of (38) with Dirichlet boundary data on $\tilde{B}$.

We come back to the proof of Theorem 8. By our remarks previous to the proof of Lemma 5 we know that at each point $p \in \partial C$, there is a ball $\tilde{B}_{p}$ of radius $\tilde{R}$ such that $\tilde{B}_{p} \subseteq C$ and $p \in \partial \tilde{B}_{p}$. Since the solution $u^{\epsilon}$ of (36) in $C$ satisfies $u^{\epsilon} \geq 0$ in $\tilde{B}_{p}$, by applying the comparison principle for the problem (38) in $\tilde{B}_{p}$ instead of $\tilde{B}$ (see Subsection 2.5) we deduce that $u^{\epsilon} \geq u_{\tilde{B}_{p}} \geq \alpha$. Since this is true for all balls $\tilde{B}_{p}$, we deduce that $u^{\epsilon} \geq \alpha$ on a neighborhood of $\partial C$. Finally, by Theorem 3 in Subsection 2.5 we have $(37)$.

Proof of Theorem 5. Assume first that $C$ is a bounded strictly convex domain in $\mathbb{R}^{N}$ of class $C^{4}$. Let $u^{\epsilon}$ be the solution constructed in Theorem 8, and let $v^{\epsilon}(x)=u^{\epsilon}\left(\frac{x}{\epsilon}\right)$. We know that $v^{\epsilon}(x)$ is a solution of

$$
\operatorname{div}\left(\frac{D v}{\sqrt{1+|D v|^{2}}}\right)+\frac{\lambda}{\epsilon}(1-v)=0 \quad \text { in } \quad \epsilon C,
$$

satisfying

$$
\left[\frac{D v^{\epsilon}}{\sqrt{1+\left|D v^{\epsilon}\right|^{2}}} \cdot \nu^{\epsilon C}\right]=\operatorname{sign}\left(0-v^{\epsilon}\right)=-1 \quad \text { on } \quad \partial(\epsilon C) .
$$

Moreover, by the results of L. Simon and J. Spruck [40], since $C$ is a bounded convex domain of class $C^{4}$, we have that $v^{\epsilon} \in C^{2}(\epsilon C) \cap C(\overline{\epsilon C})$. Let us clarify this conclusion. By the result of $\mathrm{L}$. Simon and J. Spruck [40], there is a solution $w^{\epsilon} \in C^{2}(\epsilon C) \cap C(\overline{\epsilon C})$ of (46), the boundary condition being understood in the following sense:

$$
\lim _{\eta \rightarrow 0+} \frac{1}{\eta} \int_{W \cap[d(x)<\eta]}\left|T w^{\epsilon} \cdot \nabla d(x)+1\right| d x=0
$$

for each $W \subseteq \epsilon C$, where $d(x)=d(x, \partial(\epsilon C))$, and

$$
T w^{\epsilon}:=\frac{D w^{\epsilon}}{\sqrt{1+\left|D w^{\epsilon}\right|^{2}}} .
$$

Let us prove that

$$
\left[T w^{\epsilon} \cdot \nu^{\epsilon C}\right]=-1 \quad \text { on } \partial(\epsilon C) .
$$

For that, let $\varphi \in C^{1}(\overline{\epsilon C})$, and let us multiply the $\operatorname{PDE}(46)$ by $\varphi$ and integrate it on $(\epsilon C)_{\delta}:=\{x \in$ $(\epsilon C): d(x) \geq \delta\}$ for $\delta>0$ small enough. After integrating by parts, we obtain

$$
\int_{(\epsilon C)_{\delta}} f\left(w^{\epsilon}\right) \varphi d x=-\int_{(\epsilon C)_{\delta}} \operatorname{div} T w^{\epsilon} \varphi d x=\int_{(\epsilon C)_{\delta}} T w^{\epsilon} \cdot D \varphi d x-\int_{\partial(\epsilon C)_{\delta}}\left(T w^{\epsilon} \cdot \nu^{(\epsilon C)_{\delta}}\right) \varphi d x
$$


where $f\left(w^{\epsilon}\right)=\frac{\lambda}{\epsilon}\left(1-w^{\epsilon}\right)$. Let

$$
G(x)=\frac{1}{\eta} \chi_{[d<\eta]}\left|T w^{\epsilon} \cdot \nabla d(x)+1\right|
$$

Now, we observe that (48) proves that $G(x) \rightarrow 0$ in $L^{1}(W)$, hence also

$$
G(x) \varphi(x) \rightarrow 0 \quad \text { in } L^{1}(W) .
$$

Take $W=\epsilon C$. If we write

$$
\int_{\epsilon C} G(x) \varphi(x) d x=\int_{0}^{\eta} \int_{\partial(\epsilon C)_{s}} G(x) \varphi(x) d \mathcal{H}^{N-1} d s,
$$

using (51) we deduce that for some sequence $\delta_{n} \rightarrow 0$ we have

$$
\int_{\partial(\epsilon C)_{\delta_{n}}}\left|T w^{\epsilon} \cdot \nabla d(x)+1\right| \varphi(x) d \mathcal{H}^{N-1} \rightarrow 0
$$

as $\delta_{n} \rightarrow 0$. Now, we take $\delta=\delta_{n}$ and pass to the limit as $\delta \rightarrow 0$ in (50) to obtain

$$
\begin{aligned}
\int_{(\epsilon C)} f\left(w^{\epsilon}\right) \varphi d x & =\int_{(\epsilon C)} T w^{\epsilon} \cdot D \varphi d x-\int_{\partial(\epsilon C)}(-1) \varphi d x \\
& =-\int_{(\epsilon C)} \operatorname{div} T w^{\epsilon} \varphi d x+\int_{\partial(\epsilon C)}\left[T w^{\epsilon} \cdot \nu^{(\epsilon C)}\right] \varphi d x-\int_{\partial(\epsilon C)}(-1) \varphi d x
\end{aligned}
$$

Since $\operatorname{div} T w^{\epsilon}=f\left(w^{\epsilon}\right)$ the above equality implies that $\left[T w^{\epsilon} \cdot \nu^{(\epsilon C)}\right]=-1$ on $\partial(\epsilon C)$. We conclude that $w^{\epsilon}$ is a solution of (46) and, both $v^{\epsilon}$ and $w^{\epsilon}$ satisfy the same boundary condition given in (47) and (49). A uniqueness result for this equation proves that $w^{\epsilon}=v^{\epsilon}([7],[32])$. Hence $v^{\epsilon} \in C^{2}(\epsilon C) \cap C(\overline{\epsilon C})$.

Under these circumstances, by Korevaar's Theorem [30], we deduce that $v^{\epsilon}$ is concave. Hence, also $u^{\epsilon}$ is concave. Since, as $\epsilon \rightarrow 0, u^{\epsilon}$ converges to the solution $w_{\lambda}$ of

$$
\begin{array}{ll}
u-\lambda^{-1} \operatorname{div}\left(\frac{D u}{|D u|}\right)=1 & \text { in } \quad C \\
u=0 & \text { on } \partial C
\end{array}
$$

we deduce that $w_{\lambda}$ is also concave. Moreover we know that $w_{\lambda} \geq \beta>0$ (comparison with balls), but we may also deduce this lower bound from Theorem 8 and Lemma 5 (a derivation based on inequality (35)). Thus the vector field $\xi_{\lambda},\left\|\xi_{\lambda}\right\|_{\infty} \leq 1$, satisfies $\left(\xi_{\lambda}, D w_{\lambda}\right)=\left|D w_{\lambda}\right|, w_{\lambda}-\operatorname{div} \xi_{\lambda}=1$ on $C$, and $\left[\xi_{\lambda} \cdot \nu^{C}\right]=-1$. Hence, if we define $w_{\lambda}=0$ outside $C$, we have that $w_{\lambda}$ is a solution of (26) in $\mathbb{R}^{N}$. Hence $w_{\lambda}=u_{\lambda}$. We conclude that $u_{\lambda}$ is concave in $C$. We have proved Theorem 5 when $C$ is strictly convex and of class $C^{4}$.

Let us consider now the general case where $C$ is a bounded convex set of class $C^{1,1}$ with $(N-1) \mathbf{H}_{C} \leq$ $\frac{1}{R}$. Let $C_{n}$ be a sequence of bounded strictly convex sets of class $C^{4}$ such that $C \subseteq C_{n}$ and $C=\cap_{n} C_{n}$. Moreover we may assume that $(N-1) \mathbf{H}_{C_{n}} \leq \frac{1}{R_{n}}$ with $R_{n} \rightarrow R$. By the previous paragraph the solution $u_{n}$ of

$$
u-\lambda^{-1} \operatorname{div}\left(\frac{D u}{|D u|}\right)=\chi_{C_{n}} \quad \text { in } \mathbb{R}^{N}
$$

is concave. Since $u_{n}$ converges to the solution of

$$
u-\lambda^{-1} \operatorname{div}\left(\frac{D u}{|D u|}\right)=\chi_{C} \quad \text { in } \mathbb{R}^{N}
$$

we deduce that $u_{\lambda}$ is concave in $C$. The Theorem is proved. 


\subsection{A characterization of calibrable sets by its mean curvature}

Proposition 5. Let $C$ be a bounded convex subset of $\mathbb{R}^{N}$ of class $C^{1,1}$. Let $\mu_{n} \rightarrow \mu$. Let $C_{n}$ be a minimizer of $(P)_{\mu_{n}}$. Assume that $C_{n}$ is a sequence of convex sets converging to $C$, and $C_{n} \neq C$. Then $\mu \leq(N-1)\left\|\mathbf{H}_{C}\right\|_{\infty}$.

Proof. Observe that our assumptions imply that $C$ is a minimizer of $(P)_{\mu}$. We recall that the integral of the mean curvature of a convex set $K$

$$
B(K)=\int_{\partial K} \mathbf{H}_{K} d S
$$

being a multiple of a quermassintegral [39], is an additive, continuous, and monotone functional. Since $C_{n} \subseteq C$ and $(N-1) \mathbf{H}_{C_{n}}=\mu_{n}$ on $\partial C_{n} \backslash \partial C$ we may write

$$
\begin{aligned}
0 & \leq B(C)-B\left(C_{n}\right)=\int_{\partial C} \mathbf{H}_{C} d S-\int_{\partial C_{n}} \mathbf{H}_{C_{n}} d S=\int_{\partial C \backslash \partial C_{n}} \mathbf{H}_{C} d S-\int_{\partial C_{n} \backslash \partial C} \mathbf{H}_{C_{n}} d S \\
& \leq\left\|\mathbf{H}_{C}\right\|_{\infty} \mathcal{H}^{N-1}\left(\partial C \backslash \partial C_{n}\right)-\frac{\mu_{n}}{(N-1)} \mathcal{H}^{N-1}\left(\partial C_{n} \backslash \partial C\right),
\end{aligned}
$$

hence

$$
\mu_{n} \mathcal{H}^{N-1}\left(\partial C_{n} \backslash \partial C\right) \leq(N-1)\left\|\mathbf{H}_{C}\right\|_{\infty} \mathcal{H}^{N-1}\left(\partial C \backslash \partial C_{n}\right) .
$$

Assume first that $\mathcal{H}^{N-1}\left(\partial C \backslash \partial C_{n}\right)$ does not converge to 0 as $n \rightarrow \infty$. Since $C_{n} \subseteq C$ and both sets are convex we have that $P\left(C_{n}\right) \leq P(C)$. Since

$$
0 \leq P(C)-P\left(C_{n}\right)=\mathcal{H}^{N-1}\left(\partial C \backslash \partial C_{n}\right)-\mathcal{H}^{N-1}\left(\partial C_{n} \backslash \partial C\right),
$$

and $P\left(C_{n}\right) \rightarrow P(C)$, dividing by $\mathcal{H}^{N-1}\left(\partial C \backslash \partial C_{n}\right)$, and letting $n \rightarrow \infty$, we obtain

$$
1-\frac{\mathcal{H}^{N-1}\left(\partial C_{n} \backslash \partial C\right)}{\mathcal{H}^{N-1}\left(\partial C \backslash \partial C_{n}\right)}=\frac{P(C)-P\left(C_{n}\right)}{\mathcal{H}^{N-1}\left(\partial C \backslash \partial C_{n}\right)} \rightarrow 0 .
$$

Assume now that $\mathcal{H}^{N-1}\left(\partial C \backslash \partial C_{n}\right) \rightarrow 0$. Since $C$ minimizes $(P)_{\mu}$, we have

$$
P(C)-\mu|C| \leq P\left(C_{n}\right)-\mu\left|C_{n}\right|,
$$

hence, using the isoperimetric inequality, we may write

$$
\begin{aligned}
0 \leq P(C)-P\left(C_{n}\right) & \leq \mu\left|C \backslash C_{n}\right| \leq \mu P\left(C \backslash C_{n}\right)^{N /(N-1)} \\
& \leq \mu\left(\mathcal{H}^{N-1}\left(\partial C \backslash \partial C_{n}\right)+\mathcal{H}^{N-1}\left(\partial C_{n} \backslash \partial C\right)\right)^{N /(N-1)} \\
& \leq 2^{N /(N-1)} \mu \mathcal{H}^{N-1}\left(\partial C \backslash \partial C_{n}\right)^{N /(N-1)},
\end{aligned}
$$

where the last inequality follows from the convexity of $C_{n}$. Using (54) we may write the above inequality as

$$
0 \leq \mathcal{H}^{N-1}\left(\partial C \backslash \partial C_{n}\right)-\mathcal{H}^{N-1}\left(\partial C_{n} \backslash \partial C\right) \leq 2^{N /(N-1)} \mu \mathcal{H}^{N-1}\left(\partial C \backslash \partial C_{n}\right)^{N /(N-1)} .
$$

Dividing by $\mathcal{H}^{N-1}\left(\partial C \backslash \partial C_{n}\right)$, we obtain

$$
0 \leq 1-\frac{\mathcal{H}^{N-1}\left(\partial C_{n} \backslash \partial C\right)}{\mathcal{H}^{N-1}\left(\partial C \backslash \partial C_{n}\right)} \leq 2^{N /(N-1)} \mu \mathcal{H}^{N-1}\left(\partial C \backslash \partial C_{n}\right)^{1 /(N-1)}
$$

Letting $n \rightarrow \infty$, we deduce that

$$
\frac{\mathcal{H}^{N-1}\left(\partial C_{n} \backslash \partial C\right)}{\mathcal{H}^{N-1}\left(\partial C \backslash \partial C_{n}\right)} \rightarrow 1 \quad \text { as } n \rightarrow \infty .
$$

Thus, in any case, dividing (53) by $\mathcal{H}^{N-1}\left(\partial C \backslash \partial C_{n}\right)$ and letting $n \rightarrow \infty$ we obtain that

$$
\mu \leq(N-1)\left\|\mathbf{H}_{C}\right\|_{\infty}
$$


Theorem 9. Let $C$ be a bounded convex subset of $\mathbb{R}^{N}$ of class $C^{1,1}$. Let $\Lambda:=(N-1)\left\|\mathbf{H}_{C}\right\|_{\infty}$. Let $C_{\mu}$ be the solution of $(P)_{\mu}, \mu>0$. Then $C_{\mu}=C$ if and only if $\mu \geq \max \left(\lambda_{C}, \Lambda\right)$.

Proof. Assume that $C_{\mu}=C$ is a solution of $(P)_{\mu}$. Then $(N-1) \mathbf{H}_{C} \leq \mu$ is a consequence of Proposition 1. On the other hand, $P(C)-\mu|C| \leq P(\emptyset)-\mu|\emptyset|=0$, i.e., $\mu \geq \lambda_{C}$.

Assume now that $\mu \geq \max \left(\lambda_{C}, \Lambda\right)$, but $C$ is not a minimizer of $(P)_{\mu}$. In particular, by Proposition 2 and Lemma 4.(ii), $C$ is not calibrable. We shall construct a sequence of sets $E_{\lambda} \neq C$ each one being a solution of $(P)_{\mu_{\lambda}}$ with $\mu_{\lambda} \rightarrow \beta, \beta>\mu$. Let $\lambda>\max \left(N \Lambda, \frac{1}{\left\|\chi_{C}\right\|_{*}}, \mu\right)$. By Lemma 3.(iii), we know that $u_{\lambda} \geq\left(1-N \frac{\Lambda}{\lambda}\right)^{+} \chi_{C}$. Let us define

$$
\beta_{\lambda}:=\inf \left\{\gamma: u_{\lambda} \geq\left(1-\frac{\gamma}{\lambda}\right)^{+} \chi_{C}\right\}
$$

Obviously, we have $\beta_{\lambda} \leq N \Lambda$, and

$$
u_{\lambda} \geq\left(1-\frac{\beta_{\lambda}}{\lambda}\right)^{+} \chi_{C}
$$

Case $\beta_{\lambda} \leq \mu$. Take $s=1-\frac{\mu}{\lambda}$. Then, by Proposition $4,\left[u_{\lambda} \geq s\right]$ is a solution of $(P)_{\lambda(1-s)}=(P)_{\mu}$. Finally we observe that $\left[u_{\lambda} \geq s\right]=C$. Thus $C$ is a solution of $(P)_{\mu}$. Hence, we may assume that the following case holds.

Case $\mu<\beta_{\lambda} \leq N \Lambda$. For each $\lambda>\max \left(N \Lambda, \frac{1}{\left\|\chi_{C}\right\|_{*}}\right)$, take $s_{\lambda} \in\left(1-\frac{\beta_{\lambda}}{\lambda}, 1-\frac{\beta_{\lambda}}{\lambda}+\frac{\epsilon_{\lambda}}{\lambda}\right], \epsilon_{\lambda}>0$ a sequence converging to 0 . Then

$$
\beta_{\lambda}-\epsilon_{\lambda} \leq \lambda\left(1-s_{\lambda}\right)<\beta_{\lambda} .
$$

Let $E_{\lambda}=\left[u_{\lambda} \geq s_{\lambda}\right]$. Since $\lambda\left(1-s_{\lambda}\right)<\beta_{\lambda}$, and by Lemma 3.(v), we know that $u_{\lambda}$ is not constant, by an appropriate choice of $s_{\lambda}$ we may assume that $E_{\lambda} \neq \emptyset, E_{\lambda} \neq C$. By Lemma 3.(ii), choosing $s_{\lambda}$ sufficiently near $1-\frac{\beta_{\lambda}}{\lambda}$, i.e., $\epsilon_{\lambda}$ sufficiently small, we have that $E_{\lambda} \rightarrow C$ as $\lambda \rightarrow \infty$. Without loss of generality me may assume that $\beta_{\lambda} \rightarrow \beta$ where $\mu \leq \beta \leq N \Lambda$. If $\beta=\mu$, then $\lambda\left(1-s_{\lambda}\right) \rightarrow \mu$. Since $E_{\lambda}$ is a solution of $(P)_{\lambda\left(1-s_{\lambda}\right)}$, then $C$ would be a solution of $(P)_{\mu}$, and this would conclude. Therefore we may assume that $\mu<\beta \leq N \Lambda$.

Summarizing, $E_{\lambda}$ is a solution of $(P)_{\mu_{\lambda}}$ with $\mu_{\lambda}:=\lambda\left(1-s_{\lambda}\right) \rightarrow \beta$ with $\mu<\beta \leq N \Lambda$, and $E_{\lambda} \neq C$, $E_{\lambda} \rightarrow C$.

Moreover, since $E_{\lambda}$ is an upper level set of $u_{\lambda}$ and $\lambda$ can be taken $\geq 2 N \Lambda$ (recall that $\lambda \rightarrow \infty$ ), by Theorem 5, we know that $u_{\lambda}$ is concave, hence, $E_{\lambda}$ is convex. By Proposition 5, we have that

$$
\beta \leq(N-1)\left\|\mathbf{H}_{C}\right\|_{\infty}=\Lambda \leq \mu,
$$

and we obtain a contradiction. We have proved that $C$ minimizes $(P)_{\mu}$.

Corollary 1. Let $C$ be a bounded convex subset of $\mathbb{R}^{N}$ of class $C^{1,1}$. Then $E=C$ is a solution of

$$
\min _{F \subseteq C} P(F)-\lambda_{C}|F|
$$

if and only if $(N-1) \mathbf{H}_{C} \leq \lambda_{C}$.

Remark 4. Corollary 1 extends to $\mathbb{R}^{N}$ the analogous result proved in [27] when $N=2$. From Corollary 1 and Theorem 1 in [22] (see also [19], [28]), if $(N-1) \mathbf{H}_{C} \leq \lambda_{C}$ we deduce that the capillary problem in absence of gravity can be solved for any angle $\gamma \in\left(0, \frac{\pi}{2}\right]$.

Remark 5. Observe that, if $C$ is not calibrable, then $N \Lambda \geq \frac{1}{\left\|\chi_{C}\right\|_{*}}$. Otherwise, $\left\|N \Lambda \chi_{C}\right\|_{*} \leq 1$ and there would exist a vector field $z \in L^{\infty}\left(\mathbb{R}^{N}, \mathbb{R}^{N}\right)$ with $\|z\|_{\infty} \leq 1$ such that $-\operatorname{div} z=N \Lambda \chi_{C}$. Multiplying by $\chi_{C}$ and integrating in $\mathbb{R}^{N}$ we obtain

$$
N \Lambda|C|=\int_{\mathbb{R}^{N}}\left(z, D \chi_{C}\right) \leq P(C) .
$$

Hence $\Lambda \leq N \Lambda \leq \lambda_{C}$, and $C$ would be calibrable, by Corollary 1. In particular, in the proof of the second case of Theorem 9 it suffices to take $\lambda>N \Lambda$.

Remark 6. Taking into account the regularity results of Korevaar and Simon [31], the above results can be extended to the case of crystalline norms $\phi$. This will be considered in a subsequent paper. 


\section{Convexity of the minima of the perimeter with fixed volume}

We assume that $C$ is a bounded convex set of class $C^{1,1}$.

Proposition 6. Let $\alpha \geq \frac{2 N}{R}, \lambda>\alpha\left(1-\left\|u_{\alpha}\right\|_{\infty}\right)$ where $u_{\alpha}$ denotes the solution of $(Q)_{\alpha}$. Then $(P)_{\lambda}$ has a unique solution. Moreover, the solution is convex.

Proof. Let $F$ be a minimizer of $(P)_{\lambda}$. Let us write $\lambda=\alpha(1-s)$ for some $s \in(0,1), s<\left\|u_{\alpha}\right\|_{\infty}$. Let $s_{n} \uparrow s, t_{n} \downarrow s$. Since $\alpha\left(1-s_{n}\right) \downarrow \lambda$, and $\alpha\left(1-t_{n}\right) \uparrow \lambda$, by Lemma 4.(i), we have that

$$
\cup_{n} C_{\alpha\left(1-t_{n}\right)} \subseteq F \subseteq \cap_{n} C_{\alpha\left(1-s_{n}\right)} .
$$

where $C_{\alpha\left(1-t_{n}\right)}=\left[u_{\alpha} \geq t_{n}\right], C_{\alpha\left(1-s_{n}\right)}=\left[u_{\alpha} \geq s_{n}\right]$. Finally, since $u_{\alpha}$ is concave and $s<\left\|u_{\alpha}\right\|_{\infty}$, we have that

$$
\cup_{n} C_{\alpha\left(1-t_{n}\right)}=\cap_{n} C_{\alpha\left(1-s_{n}\right)}=\left[u_{\alpha} \geq s\right] \quad \text { (modulo a null set). }
$$

Hence $F=\left[u_{\alpha} \geq s\right]$ and the Proposition follows. Being a level set of $u_{\alpha}$, the convexity of $F$ follows from the concavity of $u_{\alpha}$.

From Proposition 6 and Lemma 4.(iii) we deduce the following consequence.

Proposition 7. Let $\alpha, \beta \geq \frac{2 N}{R}$. Then $\alpha\left(1-\left\|u_{\alpha}\right\|_{\infty}\right)=\beta\left(1-\left\|u_{\beta}\right\|_{\infty}\right)$.

Proof. Assume that these two numbers are not equal. Without loss of generality, we may assume that

$$
\alpha\left(1-\left\|u_{\alpha}\right\|_{\infty}\right)<\beta\left(1-\left\|u_{\beta}\right\|_{\infty}\right) .
$$

Let us take $\lambda$ such that $\alpha\left(1-\left\|u_{\alpha}\right\|_{\infty}\right)<\lambda<\beta\left(1-\left\|u_{\beta}\right\|_{\infty}\right)$. Let us write $\lambda=\alpha(1-s)=\beta(1-t)$ for some values $s<\left\|u_{\alpha}\right\|_{\infty}$, and $t>\left\|u_{\beta}\right\|_{\infty}$. Since $\left[u_{\beta} \geq t\right]=\emptyset$, and, by Proposition 6 , the solution of $(P)_{\lambda}$ is unique, being $\left[u_{\alpha} \geq s\right]$ a solution of $(P)_{\lambda}$, we deduce that $\left[u_{\alpha} \geq s\right]=\emptyset$, a contradiction. This proves our proposition.

Let $\lambda^{*}$ be the unique value of $\alpha\left(1-\left\|u_{\alpha}\right\|_{\infty}\right)$, for $\alpha \geq \frac{2 N}{R}$, determined by the above proposition. Using Lemma 4.(iii), and Propositions 6 and 7 we obtain the following result.

Corollary 2. If $C_{\lambda}$ denotes the minimum of $(P)_{\lambda}$, the functions $\lambda \rightarrow P\left(C_{\lambda}\right)$ and $\lambda \rightarrow\left|C_{\lambda}\right|$ are continuous for $\lambda \in\left(\lambda^{*}, \infty\right)$.

Proposition 8. Let $\alpha, \beta \geq \frac{2 N}{R}$. Then $\left[u_{\alpha} \geq\left\|u_{\alpha}\right\|_{\infty}\right]=\left[u_{\beta} \geq\left\|u_{\beta}\right\|_{\infty}\right]$, and

$$
\lambda^{*}=\frac{P\left(\left[u_{\alpha} \geq\left\|u_{\alpha}\right\|_{\infty}\right]\right)}{\left|\left[u_{\alpha} \geq\left\|u_{\alpha}\right\|_{\infty}\right]\right|} .
$$

As a consequence, we obtain that this set is calibrable.

Proof. Since $\left[u_{\alpha} \geq\left\|u_{\alpha}\right\|_{\infty}-\frac{1}{\alpha n}\right]$, and $\left[u_{\beta} \geq\left\|u_{\beta}\right\|_{\infty}-\frac{1}{\beta n}\right]$ are both solutions of $(P)_{\lambda^{*}+\frac{1}{n}}$, we have that

$$
\left[u_{\alpha} \geq\left\|u_{\alpha}\right\|_{\infty}-\frac{1}{\alpha n}\right]=\left[u_{\beta} \geq\left\|u_{\beta}\right\|_{\infty}-\frac{1}{\beta n}\right]
$$

Since

$$
\left[u_{\alpha} \geq\left\|u_{\alpha}\right\|_{\infty}\right]=\cap_{n}\left[u_{\alpha} \geq\left\|u_{\alpha}\right\|_{\infty}-\frac{1}{\alpha n}\right]
$$

and

$$
\left[u_{\beta} \geq\left\|u_{\beta}\right\|_{\infty}\right]=\cap_{n}\left[u_{\beta} \geq\left\|u_{\beta}\right\|_{\infty}-\frac{1}{\beta n}\right]
$$

we deduce that $\left[u_{\alpha} \geq\left\|u_{\alpha}\right\|_{\infty}\right]=\left[u_{\beta} \geq\left\|u_{\beta}\right\|_{\infty}\right]$, and this set minimizes $(P)_{\lambda^{*}}$.

Now, since $\left[u_{\alpha} \geq\left\|u_{\alpha}\right\|_{\infty}+\epsilon\right]=\emptyset$ is a solution of $(P)_{\lambda^{*}-\lambda \epsilon}$, for all $\epsilon>0$, by Lemma 4.(iii), we have that $\emptyset$ is also a solution of $(P)_{\lambda^{*}}$. Then

$$
P\left(\left[u_{\alpha} \geq\left\|u_{\alpha}\right\|_{\infty}\right]\right)-\lambda^{*}\left|\left[u_{\alpha} \geq\left\|u_{\alpha}\right\|_{\infty}\right]\right|=P(\emptyset)-\lambda^{*}|\emptyset|=0,
$$

and (57) follows. Since $\left[u_{\alpha} \geq\left\|u_{\alpha}\right\|_{\infty}\right]$ is a minimizer of $(P)_{\lambda^{*}}$ we deduce that this set is calibrable.

Collecting the above results, we have obtained the following Theorem. 
Theorem 10. Let $C$ be a bounded convex set of class $C^{1,1}$. Then there is a convex calibrable set $K \subseteq C$. Moreover $K$ minimizes

$$
\min _{F \subseteq C} P(F)-\lambda_{K}|F|
$$

For any $\lambda>\lambda_{K}$, there is a unique minimizer $C_{\lambda}$ of $(P)_{\lambda}$ and the function $\lambda \rightarrow C_{\lambda}$ is increasing and continuous. Moreover $\lambda \rightarrow P\left(C_{\lambda}\right)$ is also continuous.

Let us state without proof the following observation.

Lemma 6. Let $C$ be a bounded convex subset of $\mathbb{R}^{N}$. Let $\mu \geq 0$ and let $E$ be a solution of the variational problem

$$
\min _{F \subseteq C} P(F)-\mu|F|
$$

Let $V=|E|$. Then $E$ is a solution of

$$
\min _{F \subseteq C,|F|=V} P(F) .
$$

Theorem 11. Let $C$ be a bounded convex set of class $C^{1,1}$. For any $V \in[|K|,|C|]$ there is a unique convex solution of the constrained isoperimetric problem (60).

Proof. Any solution of (60) corresponding to a value $V \in[|K|,|C|]$ coincides with the solution obtained from the corresponding problem $(P)_{\lambda}$ for some $\lambda \in\left[\lambda_{K}, \infty\right)$. Indeed, if $V \in[|K|,|C|]$, there is a value of $\lambda \in\left[\lambda_{K}, \infty\right)$ such that, if $C_{\lambda}$ is the minimum of $(P)_{\lambda}$, then $\left|C_{\lambda}\right|=V$. By Lemma 6 we know that $C_{\lambda}$ is a solution of (60). Now, let $Q$ be another solution of (60). We have that $P(Q)=P\left(C_{\lambda}\right)$, and $|Q|=\left|C_{\lambda}\right|$. Hence

$$
P(Q)-\lambda|Q|=P\left(C_{\lambda}\right)-\lambda\left|C_{\lambda}\right| \leq P(F)-\lambda|F|
$$

for any $F \subseteq C$. Thus, $Q$ is a minimum of $(P)_{\lambda}$, hence $Q=C_{\lambda}$.

Remark 7. By virtue of Lemma 6 and Proposition 4, the algorithm described in [16], [17], permits to compute the solution of (60) for any $V \in[|K|,|C|]$.

\section{Evolution of convex sets in $\mathbb{R}^{N}$ by the minimizing Total Variation flow}

\subsection{The evolution of a convex calibrable set}

We are interested in computing the evolution of convex sets by the minimizing Total Variation flow, i.e., the solution of the equation

$$
\left.\frac{\partial u}{\partial t}=\operatorname{div}\left(\frac{D u}{|D u|}\right) \quad \text { in } Q_{T}:=\right] 0, T\left[\times \mathbb{R}^{2},\right.
$$

coupled with the initial condition

$$
u(0)=u_{0} \in L^{2}\left(\mathbb{R}^{2}\right),
$$

when $u_{0}=\chi_{C}, C$ being a bounded convex subset of $\mathbb{R}^{N}$. Since we need the results of Section 4 we shall assume that $C$ is of class $C^{1,1}$.

Let $\Omega$ be a set of finite perimeter in $\mathbb{R}^{N}$. We shall say that the set $\Omega$ decreases at constant speed $\lambda$ if

$$
u(t, x):=(1-\lambda t)^{+} \chi_{\Omega}(x)
$$

is the strong solution of (61) and (62) corresponding to $u_{0}=\chi_{\Omega}$. It can be easily checked (see [11]) that $\Omega$ decreases at speed $\lambda$ if and only if the function $v:=\chi_{\Omega}$ satisfies the equation

$$
-\operatorname{div}\left(\frac{D v}{|D v|}\right)=\lambda v
$$


i.e., if and only if there exists a vector field $\xi \in L^{\infty}\left(\mathbb{R}^{N} ; \mathbb{R}^{N}\right)$ such that $\|\xi\|_{\infty} \leq 1$,

$$
-\operatorname{div} \xi=\lambda v
$$

and

$$
\int_{\mathbb{R}^{N}}(\xi, D v)=\int_{\mathbb{R}^{N}}|D v|
$$

In other words, the convex set decreases at constant speed if and only if it is calibrable. Now, using Theorem 9 we obtain a characterization of the bounded convex sets of class $C^{1,1}$ which decrease at constant speed.

Theorem 12. Let $C$ be a bounded convex subset of $\mathbb{R}^{N}$ of class $C^{1,1}$. The following conditions are equivalent:

(i) C decreases at constant speed.

(ii) $C$ is calibrable.

(iii) $(N-1) \sup _{p \in \partial C} \mathbf{H}_{C}(p) \leq \lambda_{C}$.

\subsection{The evolution of several convex calibrable sets with no interaction}

Let $C_{1}, \ldots, C_{m}$ be bounded convex subsets of $\mathbb{R}^{N}$ of class $C^{1,1}$ such that $\overline{C_{i}} \cap \overline{C_{j}}=\emptyset$ for any $i \neq j$, and let $F:=\mathbb{R}^{N} \backslash \bigcup_{i=1}^{m} \bar{C}_{i}$. We are concerned with the existence of a vector field $z: F \rightarrow \mathbb{R}^{N}$ such that

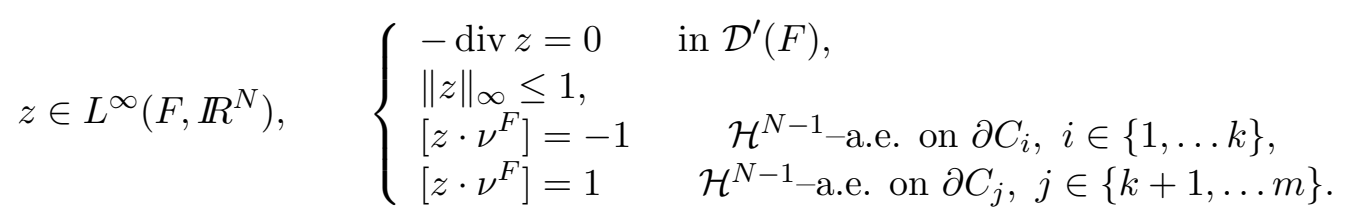

The following result was proved in [12] in $\mathbb{R}^{2}$ but the proof extends to $\mathbb{R}^{N}, N \geq 3$.

Theorem 13. The following conditions are equivalent.

(i) Problem (67) has a solution.

(ii) let $E_{1}$ be a solution of the variational problem

$$
\min \left\{P(E): \bigcup_{j=k+1}^{m} C_{j} \subseteq E \subseteq \mathbb{R}^{N} \backslash \bigcup_{i=1}^{k} C_{i}\right\}
$$

Then we have

$$
P\left(E_{1}\right)=\sum_{j=k+1}^{m} P\left(C_{j}\right) .
$$

Let $E_{2}$ be a solution of the variational problem

$$
\min \left\{P(E): \bigcup_{i=1}^{k} C_{i} \subseteq E \subseteq \mathbb{R}^{N} \backslash \bigcup_{j=k+1}^{m} C_{j}\right\}
$$

Then we have

$$
P\left(E_{2}\right)=\sum_{i=1}^{k} P\left(C_{i}\right)
$$

Moreover, if $k=0$, condition (ii) can be stated only for $E_{1}$; if $k=m$, condition (ii) can be stated only for $E_{2}$. 
Remark 8. Let $C_{1}, \ldots, C_{m}$ be bounded convex subsets of $\mathbb{R}^{N}$ of class $C^{1,1}$ such that $\overline{C_{i}} \cap \overline{C_{j}}=\emptyset$ for any $i \neq j$. The following conditions are equivalent:

(i) The following problem admits a solution:

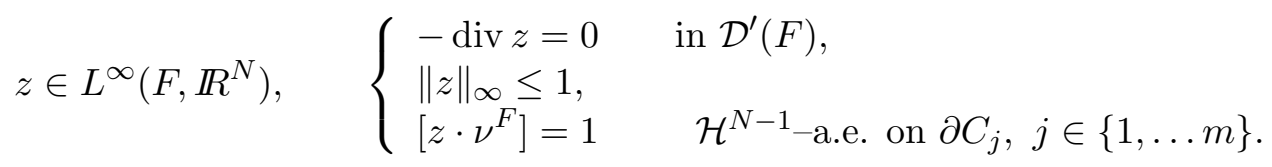

(ii) if $E_{1}$ be a solution of the variational problem

$$
\min \left\{P(E): \bigcup_{j=1}^{m} C_{j} \subseteq E\right\}
$$

then we have

$$
P\left(E_{1}\right)=\sum_{j=1}^{m} P\left(C_{j}\right)
$$

(iii) let $0 \leq k \leq m$ and let $\left\{i_{1}, \ldots, i_{k}\right\} \subseteq\{1, \ldots, m\}$ be any $k$-uple of indices; if we denote by $E_{i_{1}, \ldots, i_{k}}$ a solution of the variational problem

$$
\min \left\{P(E): \quad E \text { of finite perimeter }, \bigcup_{j=1}^{k} C_{i_{j}} \subseteq E \subseteq \mathbb{R}^{N} \backslash \bigcup_{j=k+1}^{m} C_{i_{j}}\right\}
$$

we have

$$
P\left(E_{i_{1}, \ldots, i_{k}}\right)=\sum_{j=1}^{k} P\left(C_{i_{j}}\right)
$$

Indeed, by Theorem 13, $(i)$ and (ii) are equivalent. Obviously, (iii) implies (ii). Assume now that (ii) holds. Let $E_{i_{1}, \ldots, i_{k}}$ be a minimum of (75). Let $E=E_{i_{1}, \ldots, i_{k}} \cup \bigcup_{j=k+1}^{m} C_{i_{j}}$. Then, we have

$$
P(E) \geq \sum_{j=1}^{m} P\left(C_{j}\right)
$$

Since $P(E) \leq P\left(E_{i_{1}, \ldots, i_{k}}\right)+\sum_{j=k+1}^{m} P\left(C_{i_{j}}\right)$ we deduce that

$$
P\left(E_{i_{1}, \ldots, i_{k}}\right) \geq \sum_{j=1}^{k} P\left(C_{i_{j}}\right) .
$$

Now, since $E_{i_{1}, \ldots, i_{k}}$ is a minimum of (75), we also have that

$$
P\left(E_{i_{1}, \ldots, i_{k}}\right) \leq \sum_{j=1}^{k} P\left(C_{i_{j}}\right)
$$

and we obtain (76). In particular, if one of the equivalent conditions $(i),(i i)$, or $(i i i)$ in this Remark holds, then condition (ii) of Theorem 13 also holds for all values of $k \in\{0, \ldots, m\}$. In other words, the solvability of (72) implies the solvability of $(67)$ for all values of $k \in\{0, \ldots, m\}$.

Theorem 14. Let $C_{1}, \ldots, C_{m}$ be bounded convex subsets of $\mathbb{R}^{N}$ of class $C^{1,1}$ such that $\overline{C_{i}} \cap \overline{C_{j}}=\emptyset$ for any $i \neq j$. Let $\Omega=\cup_{i=1}^{m} C_{i}$. Then $v:=\chi_{\Omega}$ is a solution of (64) if and only if 
(i) the following inequalities hold:

$$
\operatorname{ess} \sup _{p \in \partial C_{i}} \mathbf{H}_{C_{i}}(p) \leq \frac{P\left(C_{i}\right)}{\left|C_{i}\right|} \quad \forall i=1, \ldots, m
$$

(ii) $\frac{P\left(C_{i}\right)}{\left|C_{i}\right|}=\frac{P\left(C_{j}\right)}{\left|C_{j}\right|}$ for any $i, j \in\{1, \ldots, m\}$;

(iii) If $E_{1}$ is a solution of the variational problem

$$
\min \left\{P(E): \bigcup_{j=1}^{m} C_{j} \subseteq E\right\},
$$

then we have

$$
P\left(E_{1}\right)=\sum_{j=1}^{m} P\left(C_{j}\right) .
$$

Proof. Assume that $\chi_{\Omega}$ is a solution of (64). Then each set $C_{i}$ is calibrable. By Theorem 9, condition (i) holds. Now, integrating (64) on each $C_{i}$ we obtain

$$
\lambda_{\Omega}=\lambda_{C_{i}} \quad \forall i \in\{1, \ldots, m\} .
$$

Finally, we observe that, since $\chi_{\Omega}$ is a solution of (64), then there is a solution of (72). Thus, by Remark 8.(ii), we obtain that (iii) of the Theorem holds.

Conversely, assume that $(i)-($ iii $)$ hold. Let us write $\lambda_{\Omega}=\lambda_{C_{i}}, i=1, \ldots, m$. Then, by Theorem 9 , on each $C_{i}$ there is a vector field $\xi_{i}$ such that

$$
-\operatorname{div} \xi_{i}=\lambda_{\Omega} \quad \text { on } C_{i}
$$

and $\left[\xi_{i} \cdot{ }^{C_{i}}\right]=-1$ on $\partial C_{i}$. By $(i i i)$, there exists a vector field $\xi_{0}$ such that

$$
-\operatorname{div} \xi_{0}=0
$$

and $\left[\xi_{0} \cdot \nu^{C_{i}}\right]=-1$ on $\partial C_{i}$. We define

$$
\xi:= \begin{cases}\xi_{i} & \text { on } C_{i}, i \in\{1, \ldots, m\} \\ \xi_{0} & \text { on } \mathbb{R}^{N} \backslash \cup_{i=1}^{m} C_{i}\end{cases}
$$

We have

$$
-\operatorname{div} \xi=\lambda_{\Omega} \chi_{\Omega}
$$

and $\left(\xi, D \chi_{\Omega}\right)=\left|D \chi_{\Omega}\right|$. We deduce that $\chi_{\Omega}$ is a solution of (64).

Theorem 15. Let $C_{1}, \ldots, C_{m}$ be bounded convex subsets of $\mathbb{R}^{N}$ of class $C^{1,1}$ such that $\overline{C_{i}} \cap \overline{C_{j}}=\emptyset$ for any $i \neq j$. Let $b_{i}>0, i=1, \ldots, m, k \in\{1, \ldots, m\}$. Then $v:=-\sum_{i=1}^{k} b_{i} \chi_{C_{i}}+\sum_{i=k+1}^{m} b_{i} \chi_{C_{i}}$ is $a$ solution of

$$
-\operatorname{div}\left(\frac{D v}{|D v|}\right)=v
$$

if and only if

(i) the following inequalities hold:

$$
\text { ess } \sup _{p \in \partial C_{i}} \mathbf{H}_{C_{i}}(p) \leq \frac{P\left(C_{i}\right)}{\left|C_{i}\right|} \quad \forall i=1, \ldots, m ;
$$

(ii) $b_{i}=\frac{P\left(C_{i}\right)}{\left|C_{i}\right|}$ for any $i \in\{1, \ldots, m\}$; 
(iii) If $E_{1}$ is a solution of the variational problem (68), then (69) holds. If $E_{2}$ is a solution of (70), then (71) holds.

Proof. Let us write $\Omega=\cup_{i=1}^{m} C_{i}$. Assume that $v$ is a solution of (79). Let $\xi \in L^{\infty}\left(\mathbb{R}^{N}, \mathbb{R}^{N}\right)$, $\|\xi\|_{\infty} \leq 1$, be such that

$$
-\operatorname{div} \xi=v \quad \text { on } \mathbb{R}^{N}
$$

and $(\xi, D v)=|D v|$. For $i \in\{1, \ldots, k\}$, we have

$$
-\operatorname{div} \xi=-b_{i} \quad \text { on } C_{i}
$$

and $\left[\xi \cdot{ }^{C_{i}}\right]=1$ on $\partial C_{i}$. Integrating the above equation in $C_{i}$ we deduce that $b_{i}=\frac{P\left(C_{i}\right)}{\left|C_{i}\right|}$, and $C_{i}$ is calibrable, $i \in\{1, \ldots, k\}$. Similarly, since for $i \in\{k+1, \ldots, m\}$, we have

$$
-\operatorname{div} \xi=b_{i} \quad \text { on } C_{i}
$$

and $\left[\xi \cdot \nu^{C_{i}}\right]=-1$ on $\partial C_{i}$, we also deduce that $C_{i}$ are calibrable and $b_{i}=\frac{P\left(C_{i}\right)}{\left|C_{i}\right|}, i \in\{k+1, \ldots, m\}$. Then $(i)$ and (ii) hold. Finally, we observe that $\left.\xi\right|_{\mathbb{R}^{N} \backslash \Omega}$ is a solution of (67). Hence, by Theorem 13, (iii) holds.

Assume now that $(i)-(i i i)$ hold. By $(i)$ and $(i i)$, we know that there are solutions $\xi_{i}$ of $(81)$ such that $\left[\xi \cdot \nu^{C_{i}}\right]=1$ on $\partial C_{i}, i \in\{1, \ldots, k\}$. Similarly, there are solutions $\xi_{i}$ of $(82)$ such that $\left[\xi \cdot \nu^{C_{i}}\right]=-1$ on $\partial C_{i}, i \in\{k+1, \ldots, m\}$. Now, by $(i i i)$, we know that there is a solution of (67). By pasting all these solutions we find a vector field $\xi \in L^{\infty}\left(\mathbb{R}^{N}, \mathbb{R}^{N}\right),\|\xi\|_{\infty} \leq 1$, satisfying (80) and $(\xi, D v)=|D v|$.

As a consequence we obtain the following result.

Theorem 16. Let $C_{1}, \ldots, C_{m}$ be bounded convex subsets of $\mathbb{R}^{N}$ of class $C^{1,1}$ such that $\overline{C_{i}} \cap \overline{C_{j}}=\emptyset$ for any $i \neq j$. Assume that $C_{i}$ satisfy the assumptions $(i)-\left(\right.$ iii) of Theorem 15. Let $u_{0}=\sum_{i=1}^{m} b_{i} \chi_{C_{i}}$, $b_{i} \in \mathbb{R}$. Then the solution $u(t)$ of (61) corresponding to the initial condition $u(0)=u_{0}$ is $u(t)=$ $\sum_{i=1}^{m} \operatorname{sign}\left(b_{i}\right)\left(\left|b_{i}\right|-\lambda_{C_{i}} t\right)^{+} \chi_{C_{i}}$.

\subsection{The evolution of a general convex set of class $C^{1,1}$}

In this Section we assume that $C$ is a bounded convex set of class $C^{1,1}$. Let $K$ be the calibrable set contained in $C$ defined in Theorem 10. For each $\lambda \in(0, \infty)$ let $C_{\lambda}$ be the solution of $(P)_{\lambda}$. We take $C_{\lambda}=\emptyset$ for any $\lambda<\lambda_{K}$, and, by Theorem 9 we have that $C_{\lambda}=C$ for any $\lambda \geq \max \left(\lambda_{C}, \Lambda\right)$. Following the approach in [10],[29], using the monotonicity of $C_{\lambda}$ and $\left|C \backslash \cup\left\{C_{\lambda}: \lambda>0\right\}\right|=0$, we may define

$$
H_{C}(x)= \begin{cases}-\inf \left\{\lambda: x \in C_{\lambda}\right\} & \text { on } x \in C \\ 0 & \text { on } \mathbb{R}^{N} \backslash C .\end{cases}
$$

Observe that $H_{C}(x)=-\lambda_{K}$ for any $x \in K$.

Definition 3. Let $H \in L^{1}\left(\mathbb{R}^{N}\right)$. Let $\mathcal{F}_{H}$ be the functional

$$
\mathcal{F}_{H}(X)=P(X)+\int_{X} H_{C}(x) d x
$$

$X$ being a set of finite perimeter in $\mathbb{R}^{N}$. Let $E$ be a set of finite perimeter in $\mathbb{R}^{N}$. We say that $H$ is a variational mean curvature of $E$ if

$$
\mathcal{F}_{H}(E) \leq \mathcal{F}_{H}(X) \quad \forall X \text { set of finite perimeter in } \mathbb{R}^{N} .
$$

The following Proposition was proved in [10],[29].

Proposition 9. We have 
(i) $H_{C}$ is a variational mean curvature of $C$. Moreover $\left\|H_{C}\right\|_{L^{1}(C)}=P(C)$.

(ii) $H_{C} \chi_{C_{\lambda}}$ is a variational mean curvature of $C_{\lambda}$ and $\int_{C_{\lambda}} H_{C}(x) d x=-P\left(C_{\lambda}\right)$.

Lemma 7. We have $\left\|H_{C}\right\|_{*}=1$. In particular, there exists a vector field $\xi_{C} \in L^{\infty}\left(\mathbb{R}^{N}, \mathbb{R}^{N}\right)$, $\left\|\xi_{C}\right\|_{\infty} \leq 1$ such that div $\xi_{C}=H_{C}$ in $\mathbb{R}^{N}$. Moreover

$$
\left(\xi_{C}, D \chi_{C_{\lambda}}\right)=\left|D \chi_{C_{\lambda}}\right| \quad \text { for any } \lambda>0 .
$$

Proof. Since $\mathcal{F}_{H}(C)=0$, we have that $-\int_{X} H_{C}(x) d x \leq P(X)$ for any rectifiable set $X \subseteq \mathbb{R}^{N}$. As in the proof of Lemma 2 this implies that $\left\|H_{C}\right\|_{*} \leq 1$. Since $\int_{C} H_{C}(x) d x=-P(C)$ we deduce that $\left\|H_{C}\right\|_{*}=1$. Hence, by Lemma 2, there exists a vector field $\xi_{C}$ satisfying the properties of the statement of the Lemma.

Now, multiplying $\operatorname{div} \xi_{C}=H_{C}$ by $\chi_{C_{\lambda}}$ and integrating in $\mathbb{R}^{N}$ we deduce that

$$
-\int_{\mathbb{R}^{N}}\left(\xi_{C}, D \chi_{C_{\lambda}}\right)=\int_{C_{\lambda}} H_{C}(x) d x=-P\left(C_{\lambda}\right)=-\int_{\mathbb{R}^{N}}\left|D \chi_{C_{\lambda}}\right| .
$$

Since $\left\|\xi_{C}\right\|_{\infty} \leq 1$, we deduce that $\left(\xi_{C}, D \chi_{C_{\lambda}}\right)=\left|D \chi_{C_{\lambda}}\right|$.

Theorem 17. Let $C$ be a bounded convex subset of $\mathbb{R}^{N}$ of class $C^{1,1}$. Let $H_{C}(x)$ be the variational curvature of $C$ constructed in Theorem 9. Then $u(t, x)=\left(1+H_{C}(x) t\right)^{+} \chi_{C}(x)$ is the solution of (61) corresponding to the initial condition $u(0, x)=\chi_{C}(x)$.

Proof. Let $t>0$. We have $u_{t}(t, x)=\operatorname{sign}^{+}\left(1+H_{C}(x) t\right) H_{C}(x)$. Now, observe that $\operatorname{sign}^{+}(1+$ $\left.H_{C}(x) t\right)=1$ if and only if $t \leq-\frac{1}{H_{C}(x)}$, i.e., if and only if $x \in C_{1 / t}$. Otherwise $\operatorname{sign}^{+}\left(1+H_{C}(x) t\right)=0$. In particular, we observe that for $t \geq\left\|-\frac{1}{H_{C}}\right\|_{L^{\infty}(C)}=\frac{1}{\lambda_{K}}$ we have $u_{t}=0$ and also $u(t)=0$. Thus

$$
u_{t}(t, x)=H_{C}(x) \chi_{C_{1 / t}}(x) \chi_{[0, T)}(t)
$$

where $T:=\frac{1}{\lambda_{K}}$. Let $\xi_{C}$ be the vector field given by Lemma 7 . In particular, we have $\left(\xi_{C}, D \chi_{C_{1 / s}}\right)=$ $\left|D \chi_{C_{1 / s}}\right|$ for almost all $s$. In other words, we have

$$
\left[\xi_{C} \cdot \nu^{C_{1 / s}}\right]=-1 \quad \text { on } \partial C_{1 / s}
$$

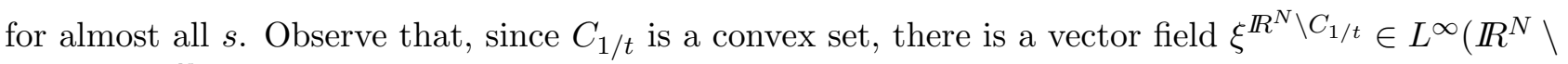
$\left.C_{1 / t}\right),\left\|\xi^{\mathbb{R}^{N} \backslash C_{1 / t}}\right\|_{\infty} \leq 1$ such that

$$
\begin{aligned}
& \operatorname{div} \xi^{\mathbb{R}^{N} \backslash C_{1 / t}}=0 \quad \text { in } \mathbb{R}^{N} \backslash C_{1 / t} \\
& {\left[\xi^{\mathbb{R}^{N} \backslash C_{1 / t}} \cdot \nu^{\mathbb{R}^{N} \backslash C_{1 / t}}\right]=1 \quad \text { on } \partial C_{1 / t} .}
\end{aligned}
$$

When $t \leq \frac{1}{\lambda_{K}}$, let

$$
\xi(t, x)=\left\{\begin{array}{ccc}
\xi_{C}(x) & \text { if } & x \in C_{1 / t} \\
\xi^{\mathbb{R}^{N} \backslash C_{1 / t}(x)} & \text { if } & x \in \mathbb{R}^{N} \backslash C_{1 / t} .
\end{array}\right.
$$


When $t \geq \frac{1}{\lambda_{K}}$, let $\xi(t, x)=0$. Let $t \leq \frac{1}{\lambda_{K}}$. By Remark 1 we have that $\xi(t) \in X_{2}\left(\mathbb{R}^{N}\right)$. We have

$$
\begin{aligned}
\int_{\mathbb{R}^{N}}(\xi(t), D u(t)) & =\int_{\mathbb{R}^{N}} \int_{0}^{\infty}\left(\xi(t), D \chi_{[u(t) \geq \lambda]}\right) d \lambda=\int_{0}^{\infty} \int_{\mathbb{R}^{N}}\left(\xi(t), D \chi_{[u(t) \geq \lambda]}\right) d \lambda \\
& =\int_{0}^{\|u(t)\|_{\infty}} \int_{\mathbb{R}^{N}}\left(\xi(t), D \chi_{[u(t) \geq \lambda]}\right) d \lambda \\
& =-\int_{0}^{\|u(t)\|_{\infty}} \int_{\partial^{*}[u(t) \geq \lambda]}\left[\xi(t) \cdot \nu^{[u(t) \geq \lambda]}\right] d \lambda, \\
& =-\int_{0}^{\|u(t)\|_{\infty}} \int_{\partial^{*}[u(t) \geq \lambda]}\left[\xi(t) \cdot \nu^{\left.C_{(1-\lambda) / t}\right] d \lambda,}\right. \\
& =-\int_{0}^{\|u(t)\|_{\infty}} \int_{\partial^{*}[u(t) \geq \lambda]}\left[\xi C(t) \cdot \nu^{\left.C_{(1-\lambda) / t}\right]} d \lambda,\right. \\
& =\int_{0}^{\|u(t)\|_{\infty}} P\left(\partial^{*}[u(t) \geq \lambda]\right) d \lambda \quad(\text { by }(84)) \\
& =\int_{\mathbb{R}^{N}}^{|D u(t)| .}
\end{aligned}
$$

Also, for $t \geq \frac{1}{\lambda_{K}}$, we have $(\xi(t), D u(t))=|D u(t)|$.

On the other hand, by construction of $\xi(t, x)$ we have

$$
\operatorname{div} \xi(t)=H_{C}(x) \chi_{C_{1 / t}}(x)
$$

if $t \leq \frac{1}{\lambda_{K}}$. If $t>\frac{1}{\lambda_{K}}$, we have $\operatorname{div} \xi(t)=0$. Thus we have that $u_{t}(t)=\operatorname{div} \xi(t)$ for almost all $t \in(0, T)$ (also in $\mathcal{D}^{\prime}\left((0, T) \times \mathbb{R}^{N}\right)$ for any $\left.T>0\right)$. By the characterization of $\partial \Psi$ given in Lemma 1 we have that $u(t)$ is a strong solution in the sense of semigroups of (61). Finally, by Theorem $2, u$ is also the strong solution of $(61)$ corresponding to the initial condition $u(0, x)=\chi_{C}(x)$.

\subsection{Solutions constructed from convex sets}

Theorem 18. Let $C_{1}, \ldots, C_{m}$ be bounded convex subsets of $\mathbb{R}^{N}$ of class $C^{1,1}$ such that $\overline{C_{i}} \cap \overline{C_{j}}=\emptyset$ for any $i \neq j$. Assume that $C_{i}$ satisfy condition (ii) in Remark (8). Let $H_{C_{i}}$ be the variational curvature of $C_{i}$ defined in Subsection 5.3. Let $b_{i} \in \mathbb{R}, b_{i}<0, i=1, \ldots, k, b_{i}>0, i=k+1, \ldots, m$. Then $u(t, x)=\sum_{i=1}^{m} \operatorname{sign}\left(b_{i}\right)\left(\left|b_{i}\right|+H_{C_{i}}(x) t\right)^{+} \chi_{C_{i}}(x)$ is the solution of (61) corresponding to the initial condition $u(0, x)=\sum_{i=1}^{m} b_{i} \chi_{C_{i}}$.

Proof. By Lemma 7 , for each $i=1, \ldots, m$, there is a vector field $\xi_{C_{i}} \in L^{\infty}\left(\mathbb{R}^{N}, \mathbb{R}^{N}\right),\left\|\xi_{C_{i}}\right\|_{\infty} \leq 1$, such that

$$
\operatorname{div} \xi_{C_{i}}=H_{C_{i}} \quad \text { in } \mathbb{R}^{N}
$$

and

$$
\int_{\mathbb{R}^{N}}\left(\xi_{C_{i}}, D \chi_{C_{i, \lambda}}\right)=\int_{\mathbb{R}^{N}}\left|D \chi_{C_{i, \lambda}}\right| \text { for any } \lambda>0 .
$$

Observe that this equality implies that

$$
\left[\xi_{C_{i}} \cdot \nu^{C_{i}}\right]=-1 \quad \mathcal{H}^{N-1} \text {-a.e. on } \partial C_{i} .
$$

Now, since $C_{i}$ satisfy condition (ii) in Remark (8), there is a vector field $\xi_{e} \in L^{\infty}\left(\mathbb{R}^{N} \backslash\left(C_{1} \cup \ldots C_{m}\right)\right)$ with $\left\|\xi_{e}\right\|_{\infty} \leq 1$ such that

$$
\left\{\begin{array}{lr}
-\operatorname{div} \xi_{e}=0 & \text { in } \mathcal{D}^{\prime}\left(\mathbb{R}^{N} \backslash \bigcup_{i=1}^{m} \overline{C_{i}}\right), \\
{\left[\xi_{e} \cdot \nu^{C_{i}}\right]=-1} & \mathcal{H}^{N-1} \text {-a.e. on } \partial C_{i}, i \in\{1, \ldots, m\}
\end{array}\right.
$$


Let us put together all these vector fields, i.e., let

$$
\xi(x)=\left\{\begin{array}{ccc}
\xi_{C_{i}}(x) & \text { if } & x \in C_{i} \\
\xi_{e}(x) & \text { if } & x \in \mathbb{R}^{N} \backslash\left(C_{1} \cup \ldots \cup C_{m}\right) .
\end{array}\right.
$$

Since $\left[\xi_{e} \cdot \nu^{C_{i}}\right]=\left[\xi_{C_{i}} \cdot \nu^{C_{i}}\right]=-1 \mathcal{H}^{N-1}$-a.e. on $\partial C_{i}$, we have that

$$
-\operatorname{div} \xi=-\sum_{i=1}^{m} H_{C_{i}} \chi_{C_{i}} \quad \text { in } \mathbb{R}^{N}
$$

Let $F=-\sum_{i=1}^{m} H_{C_{i}} \chi_{C_{i}}=\sum_{i=1}^{m}\left|H_{C_{i}}\right| \chi_{C_{i}} \geq 0$. The inequality (87) says that $\|F\|_{*} \leq 1$. We have that

$$
0 \leq \sum_{i=1}^{m}\left|H_{C_{i}}\right| \chi_{C_{i, 1 / s}} \leq \sum_{i=1}^{m}\left|H_{C_{i}}\right| \chi_{C_{i}}
$$

hence

$$
\left\|\sum_{i=1}^{m}\left|H_{C_{i}}\right| \chi_{C_{i, 1 / s}}\right\|_{*} \leq\left\|\sum_{i=1}^{m}\left|H_{C_{i}}\right| \chi_{C_{i}}\right\|_{*} \leq 1 .
$$

By Lemma 2, we conclude that there exists a vector field $\xi_{s} \in L^{\infty}\left(\mathbb{R}^{N}, \mathbb{R}^{N}\right)$ such that $\left\|\xi_{s}\right\|_{\infty} \leq 1$ such that

$$
-\operatorname{div} \xi_{s}=\sum_{i=1}^{m}\left|H_{C_{i}}\right| \chi_{C_{i, 1 / s}}
$$

and this implies that

$$
\operatorname{div} \xi_{s}=0 \quad \text { in } \mathbb{R}^{N} \backslash\left(C_{1,1 / s} \cup \ldots \cup C_{m, 1 / s}\right),
$$

and

$$
\left[\xi_{s} \cdot \nu^{C_{i, 1 / s}}\right]=-1 \quad \mathcal{H}^{N-1} \text {-a.e. on } \partial C_{i, 1 / s}, i \in\{1, \ldots, m\} .
$$

Let $I^{+}:=\left\{i: b_{i} \geq 0\right\}, I^{-}:=\left\{i: b_{i}<0\right\}$. By Remark 8 we know that there is a solution there is a vector field $\xi_{s}^{*} \in L^{\infty}\left(\mathbb{R}^{N}, \mathbb{R}^{N}\right),\left\|\xi_{s}^{*}\right\|_{\infty} \leq 1$ satisfying

$$
\left\{\begin{array}{lr}
-\operatorname{div} \xi_{s}^{*}=0 & \text { in } \mathcal{D}^{\prime}\left(\mathbb{R}^{N} \backslash \bigcup_{i=1}^{m} \overline{C_{i, 1 / s}}\right), \\
{\left[\xi_{s}^{*} \cdot \nu^{C_{i, 1 / s}}\right]=-1} & \mathcal{H}^{N-1}-\text { a.e. on } \partial C_{i, 1 / s}, i \in I^{+}, \\
{\left[\xi_{s}^{*} \cdot \nu^{C_{i, 1 / s}}\right]=1} & \mathcal{H}^{N-1} \text {-a.e. on } \partial C_{j, 1 / s}, j \in I^{-} .
\end{array}\right.
$$

Now we finish the proof as in Theorem 17, the vector field $\xi_{s}^{*}$ playing the role of the vector field $\xi^{\mathbb{R}^{N} \backslash C_{1 / t}}$ in the proof of that Theorem.

Acknowledgement. The second author acknowledges partial support by the Departament d'Universitats, Recerca i Societat de la Informació de la Generalitat de Catalunya and by PNPGC project, reference BFM2000-0962-C02-01.

\section{References}

[1] F. Alter, V. Caselles, and A. Chambolle, Evolution of Convex Sets in the Plane by the Minimizing Total Variation Flow, Preprint 2003.

[2] L. Ambrosio, Corso introduttivo alla teoria geometrica della misura ed alle supefici minime, Scuola Normale Superiore di Pisa, 1997.

[3] L. Ambrosio, Minimizing movements, Rend. Accad. Naz. Sci. XL Mem. Mat. Appl. (5) 19, 191-246, 1995. 
[4] L. Ambrosio, N. Fusco, and D. Pallara, Functions of Bounded Variation and Free Discontinuity Problems. Oxford Mathematical Monographs, 2000.

[5] F. Andreu, C. Ballester, V. Caselles, and J.M. Mazón, Minimizing total variational flow. Diff. Int. Eqs., vol. 4(3), pp. 321-360, 2001.

[6] F. Andreu, C. Ballester, V. Caselles, and J.M. Mazón, The Dirichlet Problem for the Total Variation Flow Journal Functional Analsysis, vol. 180, pp. 347-403, 2001.

[7] F. Andreu, V. Caselles, and J.M. Mazón, A parabolic quasilinear problem for linear growth functionals. Rev. Mat. Iberoamericana 18 (2002), 135-185.

[8] G. Anzellotti, Pairings between measures and bounded functions and compensated compactness. Ann. Mat. Pura Appl., 135:293-318, 1983.

[9] F.V. Atkinson, and L.A. Peletier, Bounds for vertical points of solutions of prescribed mean curvature type equations, Proceedings of the Royal Society of Edinburgh, 112A (1989), 15-32.

[10] E. Barozzi, The curvature of a boundary with finite area, Atti Accad. Naz. Lincei Cl. Sci. Fis. Mat. Natur. Rend. Lincei (9) Mat. Appl. 5 (1994), 149-159.

[11] G. Bellettini, V. Caselles and M. Novaga, The Total Variation Flow in $\mathbb{R}^{N}$, J. Differential Equations, 184:475-525, 2002.

[12] G. Bellettini, V. Caselles and M. Novaga, Explicit solutions of the eigenvalue problem $-\operatorname{div}\left(\frac{D u}{|D u|}\right)=u$, Preprint, 2003.

[13] G. Bellettini, M. Novaga, and M. Paolini, On a crystalline variational problem. II. BV regularity and structure of minimizers on facets, Arch. Ration. Mech. Anal. 157(3), 193-217, 2001.

[14] G. Bellettini, M. Novaga, and M. Paolini, Characterization of facet-breaking for nonsmooth mean curvature flow in the convex case. Interfaces Free Bound., 3:415-446, 2001.

[15] H. Brezis, Operateurs Maximaux Monotones. North Holland, 1973.

[16] A. Chambolle, An algorithm for total variation minimization and applications. Preprint CEREMADE 0240, 2002

[17] A. Chambolle, An algorithm for mean curvature motion, Preprint, Ceremade, 2003.

[18] J.T. Chen, On the existence of capillary free surfaces in the absence of gravity. Pacific J. Math., 88:323-361, 1980 .

[19] P. Concus and R. Finn, On Capillary Free Surfaces in the Absence of Gravity, Acta Mathematica, 132, 177-198, 1974.

[20] M.G. Crandall and T.M. Liggett, Generation of semigroups of nonlinear transformations on general Banach spaces. Amer. J. Math., 93:265-298, 1971.

[21] L.C. Evans and R. F. Gariepy, Measure Theory and Fine Properties of Functions. Studies in Advanced Math., CRC Press, Ann Harbor, 1992.

[22] R. Finn, Existence and non existence of capillary surfaces, Manuscripta Mathematica, 28, 1-11, 1979.

[23] R. Finn, A subsidiary variational problem and existence criteria for capillary surfaces. J. Reine Angew. Mathematik, 353:196-214, 1984.

[24] R. Finn, Equilibrium Capillary Surfaces. Springer Verlag, 1986. 
[25] M.H. Giga, Y. Giga, and R. Kobayashi, Very singular diffusion equations. Proc. of Taniguchi Conf. on Math., Advanced Studies in Pure Mathematics, 31, 93-125, 2001.

[26] Y. Giga, Singular diffusivity-facets, shocks and more. Hokkaido University Preprint Series in Mathematics, Series 604, September 2003.

[27] E. Giusti, On the equation of surfaces of prescribed mean curvature. Existence and uniqueness without boundary conditions. Invent. Math., 46:111-137, 1978.

[28] E. Giusti, Boundary Value Problems for Non-Parametric Surfaces of Prescribed Mean Curvature, Ann. Sc. Norm. Sup. Pisa (4) 3, pp. 501-548, 1976.

[29] E. Gonzalez and U. Massari, Variational Mean Curvatures, Rend. Sem. Mat. Univ. Pol. Torino, 52(1), pp. 1-28, 1994.

[30] N. Korevaar, Capillary surface convexity above convex domains, Indiana Univ. Math. Journal, 32 (1983), 73-82.

[31] N. Korevaar and L. Simon, Equations of mean curvature type with contact angle boundary conditions, Geometric analysis and the calculus of variations, pp. 175-201, Internat. Press, Cambridge, MA, 1996.

[32] A. Lichnewski and R. Temam, Pseudosolutions of the Time Dependent Minimal Surface Problem, Journal of Differential Equations 30 (1978), 340-364.

[33] U. Massari, Frontiere orientate di curvatura media assegnata in $L^{p}$. Rend. Sem. Mat. Univ. Padova, 53, pp. 37-52, 1975.

[34] Y. Meyer, Oscillating patterns in image processing and nonlinear evolution equations, The fifteenth Dean Jacqueline B. Lewis memorial lectures. University Lecture Series, 22. American Mathematical Society, Providence, RI, 2001.

[35] M. Miranda, Un principio di massimo forte per le frontiere minimali e una sua applicazione alla risoluzione del problema al contorno per l'equazione delle superfici di area minima. Rend. Sem. Mat. Univ. Padova, XLV, pp. 355-366, 1971.

[36] C. Rosales, Isoperimetric regions in rotationally symmetric convex bodies, Indiana University Math. Journal, to appear.

[37] L. Rudin, S. Osher, and E. Fatemi, Nonlinear total variation based noise removal algorithms. Physica D, 60, pp. 259-268, 1992.

[38] L.A. Santaló, Integral geometry and geometric probability. Encyclopedia of Mathematics and its Applications, Vol. 1. Addison-Wesley Publishing Co., Reading, Mass.-London-Amsterdam, 1976.

[39] R. Schneider, Convex Bodies: The Brunn-Minkowski Theory, Encyclopedia of Mathematics and its Applications, 44, Cambridge University Press, 1993.

[40] L. Simon and J. Spruck, Existence and Regularity of a Capillary Surface with Prescribed Contact Angle, Arch. Rational Mech. Anal. 61, pp. 19-34, 1976.

[41] E. Stredulinsky, and W.P. Ziemer, Area Minimizing Sets Subject to a Volume Constraint in a Convex Set. J. Geom. Anal. 7, pp. 653-677, 1997.

[42] W.P. Ziemer, Weakly Differentiable Functions. Springer Verlag, Ann Harbor, 1989. 